\title{
Three-Dimensional Cubic Barcodes
}

\author{
Golnoosh Elhami, Student Member, IEEE, Adam Scholefield, Member, IEEE, and Martin Vetterli, Fellow, IEEE
}

\begin{abstract}
We consider three-dimensional cubic barcodes, consisting of smaller cubes, each built from one of two possible materials and carry one bit of information. To retrieve the information stored in the barcode, we measure a 2-D projection of the barcode using a penetrating wave such as $\mathrm{X}$-rays, either using parallel-beam or cone-beam scanners from an unknown direction. We derive a theoretical representation of this scanning process and show that for a known barcode pose with respect to the scanner, the projection operator is linear and can be easily inverted. Moreover, we provide a method to estimate the unknown pose of the barcode from a single 2-D scan. We also propose coding schemes to correct errors and ambiguities in the reconstruction process. Finally, we test our designed barcode and reconstruction algorithms with several simulations, as well as a real-world barcode acquired with an X-ray cone-beam scanner, as a proof of concept.
\end{abstract}

Index Terms-Rectangular cuboid barcodes, Cubic barcode, Reed-Solomon, Parallel-beam scanner, Cone-beam scanner, Orthogonal projection, Central projection, Noise robustness.

\section{INTRODUCTION}

One-dimensional barcodes are a keystone of modern life providing, amongst other things, a universal product code used by checkout systems the world over. More recently, twodimensional barcodes, in the form of QR codes, have gained in popularity largely thanks to the proliferation of smartphone cameras.

In this paper, we consider three-dimensional barcodes with an $N \times N \times N$ structure as depicted in Figure 1a Here, each of the $N^{3}$ cubes is made of one of two possible materials and can thus store one bit of information. To scan the barcode, we expose it to a penetrating wave, such as X-rays, light, or sound, and measure the received signal intensity on a 2$\mathrm{D}$ plane. Therefore, what we are measuring is the absorption that the wave undergoes when passing through the barcode. While there are parallels with tomography setups, we assume we measure only a single projection from an unknown angle. Figure $1 \mathrm{~b}$ depicts an example of such a projection.

The setup we have just described can be viewed as a communication channel, which raises the question of how information should be communicated across it. We propose a practical recovery algorithm and investigate its recovery properties both theoretically and practically to address this question.

We envision several possible applications. For example, consider object tracking and identification in warehouses where it is challenging to print a barcode in a specific part of an object

This work was supported by "FNS SESAM - Sensing and Sampling: Theory and Algorithms" no. 200021_181978/1.

The authors are with the School of Computer and Communication Sciences, Ecole Polytechnique Fédérale de Lausanne (EPFL), CH-1015 Lausanne, Switzerland (email: golnoosh.elhami@epfl.ch, adam.scholefield@epfl.ch, martin.vetterli@epfl.ch).

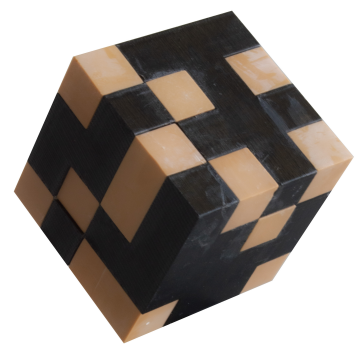

(a)

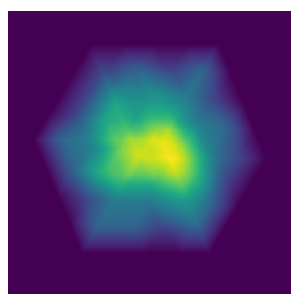

(b)
Fig. 1. (a) 3-D printed cubic barcode with 64 bits, and (b) a 2-D absorptive scan of it obtained using X-rays.

and align it with a scanning camera to read the information. However, since we can decode our barcode from a single scan taken from almost any unknown direction, we can equip each object with a 3-D cubic barcode and easily and robustly identify it. Another advantage of 3-D barcodes is that many bits are inside the code and thus not visible to non-penetrating waves. This property could be exploited in security printing applications [1], [2].

In this paper, we provide a full end-to-end analysis of our approach. First, in Section II] we derive the theoretical representation for the attenuation coefficient of cubic barcodes and the measured 2-D scan image, for both parallel-beam (orthogonal projection) and cone-beam (central projection) scanners. We show that the projection operator (forward model) is linear and straightforward to invert for a fixed barcode pose.

In Section III we show how to estimate the unknown pose of the barcode from a single 2-D projection, for both orthogonal and central projection. To do this, we use the external shape of the barcode, which we know is a cube. We do not embed in the barcode any information about its local coordinate system. Thus, the resulting pose estimation is up to an equivalence class as the barcode can be rotated 90 degrees around any of its axes, and the external shape is unchanged.

To resolve this ambiguity and uniquely identify each bit, we propose coding schemes in Section V Combined with the results of the previous two sections, this gives us a way to estimate the bits from a single projection from an unknown direction. For the coding, we adapt Reed-Solomon codes in two different manners to be applicable to a 3-D barcode and provide the advantages of each approach. As well as resolving the previously described ambiguity, the coding schemes also provide typical error correction.

In Section VI] we study the robustness of our approach to different scanning directions. To do this, we take an information theoretic approach and provide upper bounds on the information content retrievable from each scanning direction.

Finally, in Section VII. we use simulations to examine the 
robustness of our reconstruction algorithm against noise and then provide a real-world experiment that tests the whole pipeline using an X-ray cone-beam scanner.

\section{A. Related work}

The initial barcode design goes back to 1952 by Silver and Woodland [3]. This one-dimensional barcode used thin vs. thick bars to represent data similar to the Morse code. Variations of this design include KarTrak [4], Code39, Code128, and ITF14. To store more information in this visual representation, David Allais, in 1987, developed the first 2D Barcode, called Code 49 [5]. Soon after, followed Data Matrix [6], Aztec Code [7], PDF417 [8], quick response (QR) Code [9], and many other 2-D Barcodes [10], [11], [12]. One proposed approach to move beyond two-dimensional barcodes is to include color information. ColorCode [13], University of Cambridge's Cronto Visual Cryptogram [14], Microsoft's High Capacity Color Barcode (HCCB) [15], and JAB-Code [16] are all examples of barcodes with two spatial dimensions and one color dimension. While adding color information increases the capacity, it creates additional challenges, as effects such as lighting conditions, can affect the appearance of a color image [12].

In terms of barcodes with three spatial dimensions, a few different approaches have been studied. A design by Xerox proposes using elevation to encode more information in the barcode [17]. Prasad proposes using a laser to encode information in different layers of a two-photon dye [18]. Kikuchi et al. introduce a framework to print QR codes onto B-spline surfaces [19].

The main difference between these approaches and ours is that we use the additional spatial dimension to increase scanning flexibility. More precisely, our approaches require just one scan from an unknown direction.

\section{B. General setup}

In general, our setup can easily be extended to $P$ projections of a rectangular cuboid barcode, with dimensions $N_{1} \times N_{2} \times N_{3}$ consisting of cubes made of one of $M$ different materials. In this case, each voxel stores $\log _{2} M$ bits of information. We assume that the $N_{1} N_{2} N_{3}$ information bits are distributed along the third, then the second and finally the first dimension of the cube. We also do not reserve any bits to identify the local coordinate system of the barcode.

In Section III, we will show that reconstructing a cubic barcode from a single projection leads to an equivalent class of 24 barcodes that all describe the measurements. When $N_{1} \neq$ $N_{2} \neq N_{3}$, the equivalence class is reduced to 4 elements as the barcode can only rotate 180 degrees around each of its axes without changing its external shape.

In this paper, we focus on the case where $P=1$, $N_{1}=N_{2}=N_{3}=N$ and $M=2$. These restrictions simplify notation and impose clarity. Furthermore, the first two make the problem harder since the size of the equivalence class will be larger. Note that in all cases, it is easy to extend the presented techniques to the general case. In particular, since we use a linear reconstruction algorithm, it is easy to add more

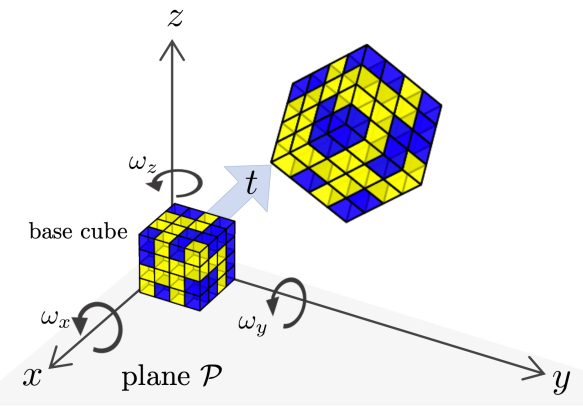

Fig. 2. Rotation and translation of the base cubic barcode.

measurements when $P>1$ by first estimating the pose of each projection using the techniques that will be present.

\section{CUBiC BARCODES Formulation}

\section{A. Barcode Formulation}

Our proposed three-dimensional cubic barcode consists of $N \times N \times N$ voxels made of two materials with substantial density value differences, each representing one bit of information. In order to retrieve the hidden information inside the barcode, we need signals that can penetrate the object. While going through the object, these signals get attenuated in proportion to the characteristics of the material. Attenuation is defined as the reduction in the intensity of a beam as it traverses the matter. According to the Beer-Lambert law [20], signal attenuation is modeled as

$$
\frac{\mathrm{d} I(l)}{\mathrm{d} l}=-\mu(l) I(l)
$$

Here, $I(l)$ is the signal intensity at depth $l$ of the material (on an axis parallel to the direction of the beam) and $\mu(l)$ is the linear attenuation coefficient with the unit of $m^{-1}$ (reciprocal meter), which depends on the characteristic of the material that the beam is passing through. Solving the differential equation in (1) results in

$$
I(l)=I_{0} e^{-\int_{0}^{l} \mu(\lambda) \mathrm{d} \lambda},
$$

where $I_{0}$ is the signal intensity at the source. In particular, if the attenuation coefficient is constant and equal to $\mu_{0}$, then we have the well-known intensity attenuation formula:

$$
I(l)=I_{0} e^{-\mu_{0} l} .
$$

We assume that each small cube inside the barcode has a side length equal to $d$ and is made of a single material with a constant attenuation coefficient of $\mu_{\text {low }}$ or $\mu_{\text {high }}$. Thus, the barcode is a cubic structure with a side length of $N d$ and varying attenuation coefficients inside. In this structure, the code-bits are defined as $b_{i j k}$ for $i, j, k \in\{0,1, \cdots, N-1\}$. Each cube is a carrier for a single code-bit assigned as

$$
\mu_{i j k}=\left\{\begin{array}{ll}
\mu_{\text {low }}, & b_{i j k}=0 \\
\mu_{\text {high }}, & b_{i j k}=1
\end{array} .\right.
$$

If a barcode is located at the origin and aligned with the axes (call it the base barcode), we can define the attenuation coefficient $\mu_{B}(\boldsymbol{x})$, at each $3-\mathrm{D}$ point $\boldsymbol{x}$, as

$$
\mu_{B}(\boldsymbol{x})=\sum_{i=0}^{N-1} \sum_{j=0}^{N-1} \sum_{k=0}^{N-1} \mu_{i j k} \psi\left(\boldsymbol{x}-d\left[\begin{array}{l}
i \\
j \\
k
\end{array}\right]\right) \text {, }
$$




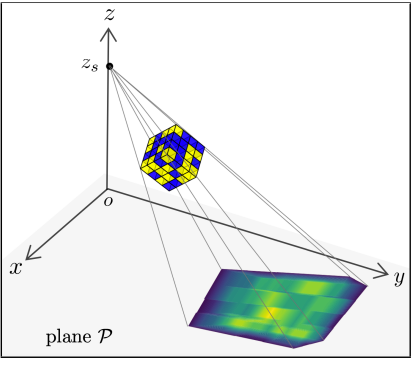

(a)

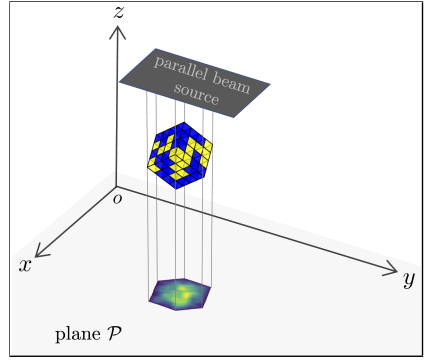

(b)

Fig. 3. (a) Central projection and (b) orthogonal projection.

where the subscript $B$ stands for 'base'. Here, we have used the basis function $\psi(\boldsymbol{x}): \mathbb{R}^{3} \rightarrow \mathbb{R}$ defined as

$$
\psi(\boldsymbol{x})=\mathbb{1}_{\left\{0 \leq x_{i} \leq d\right\}}= \begin{cases}1 & 0 \leq x_{i} \leq d \\ 0 & \text { otherwise }\end{cases}
$$

In order to simplify the notation, we change the indices of the bits in the barcode as follows:

$$
\mu_{B}(\boldsymbol{x})=\sum_{n=0}^{N^{3}-1} \mu_{n} \psi\left(\boldsymbol{x}-\boldsymbol{\tau}_{n}\right),
$$

where $\boldsymbol{\tau}_{n}=d[i, j, k]^{\top}$, and $n=i+j N+k N^{2}$.

While scanning the barcode, an image gets recorded on a scan plane $\mathcal{P}$. Without any loss of generality, we assume that the scan plane $\mathcal{P}$ is located on the $x y$-plane, and the cubic barcode has an arbitrary pose in space. In order to model the pose of the barcode in space, we assume that a base barcode defined by $\mu_{B}(\boldsymbol{x})$ in (6) is first rotated over the $x, y$ and $z$ axes with corresponding angles in $\boldsymbol{\omega}=\left[\omega_{x}, \omega_{y}, \omega_{z}\right]^{T}$, and then translated according to the translation vector $\boldsymbol{t}=$ $\left[t_{x}, t_{y}, t_{z}\right]^{T} \in \mathbb{R}^{3}$. This is shown in Figure 2 With this assumption, we can derive the attenuation coefficient of a barcode positioned arbitrarily in space as

$$
\mu(\boldsymbol{x})=\mu_{B}\left(T^{-1}(\boldsymbol{x})\right)=\sum_{n=0}^{N^{3}-1} \mu_{n} \psi\left(T^{-1}(\boldsymbol{x})-\boldsymbol{\tau}_{n}\right) .
$$

Here, $T(\boldsymbol{x})$ is the transformation that rotates and translates a given point with $\boldsymbol{\omega}$ and $t$, respectively, i.e.

$$
\left[\begin{array}{c}
T(\boldsymbol{x}) \\
1
\end{array}\right]=\boldsymbol{T}\left[\begin{array}{l}
\boldsymbol{x} \\
1
\end{array}\right]
$$

with

$$
\begin{aligned}
\boldsymbol{T}= & {\left[\begin{array}{cccc}
1 & 0 & 0 & t_{x} \\
0 & 1 & 0 & t_{y} \\
0 & 0 & 1 & t_{z} \\
0 & 0 & 0 & 1
\end{array}\right] \times\left[\begin{array}{cccc}
\cos \omega_{z} & -\sin \omega_{z} & 0 & 0 \\
\sin \omega_{z} & \cos \omega_{z} & 0 & 0 \\
0 & 0 & 1 & 0 \\
0 & 0 & 0 & 1
\end{array}\right] \times } \\
& {\left[\begin{array}{ccccc}
\cos \omega_{y} & 0 & \sin \omega_{y} & 0 \\
0 & 1 & 0 & 0 \\
-\sin \omega_{y} & 0 & \cos \omega_{y} & 0 \\
0 & 0 & 0 & 1
\end{array}\right] \times\left[\begin{array}{cccc}
1 & 0 & 0 & 0 \\
0 & \cos \omega_{x} & -\sin \omega_{x} & 0 \\
0 & \sin \omega_{x} & \cos \omega_{x} & 0 \\
0 & 0 & 0 & 1
\end{array}\right] . }
\end{aligned}
$$

In the following sections, we will use (7) to derive the intensity of the signal on the scan plane after passing through the barcode.

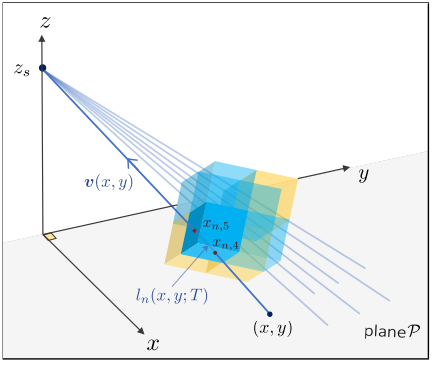

(a)

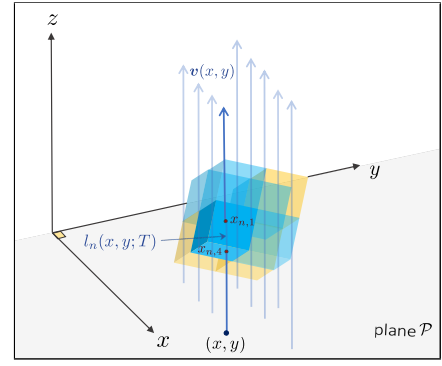

(b)
Fig. 4. (a) Central versus (b) orthogonal projection of cubic barcodes and calculation of $l_{n}(x, y ; T)$ in each case.

\section{B. Projection Types}

When we scan an object, we perform a projection of that object onto the scan plane. We will consider both orthogonal and central projections as they accurately model most practical setups. On one hand, if the scanner source can be modeled as a point source that omits cone-beams, then the projection is central. On the other hand, when the source emits parallel beams, like in medical X-ray scanners, or when the far field assumption holds, the projection is orthogonal. In Figure 3 we illustrate both the orthogonal and central projections.

Note that in the case of orthogonal projection, if we change the value of translation vector $t$, the scan is simply shifted on the image plane. Moreover, applying a rotation along the $z$ axis with angle $\omega_{z}$, results in a rotation of the scan on the scan plane $\mathcal{P}$. In the above cases, the scan values do not change (only shift or rotate).

In central projection, we assume a source $s$ is located on the $z$-axis at position $\left[0,0, z_{s}\right]^{T}$. The translation vector $\boldsymbol{t}$ does indeed change the scan values, because it changes the relative location of the barcode versus the source $s$ (see Figure 3a.

\section{Scan Formulation}

While scanning the barcode, the scanner records the intensity of the signal observed on the scan plane $\mathcal{P}$; call it $I(x, y)$. Following the Beer-Lambert law in (2), the intensity of the ray passing through the barcode and recorded on $\mathcal{P}$ can be formulated as

$$
I(x, y)=I_{0} e^{-\int_{\boldsymbol{v}(x, y)} \mu(\boldsymbol{x}) \mathrm{d} \lambda}
$$

where $\int_{\boldsymbol{v}(x, y)} \cdot \mathrm{d} \lambda: \mathbb{R}^{3} \rightarrow \mathbb{R}^{2}$ denotes integration along lines in direction $\boldsymbol{v}(x, y)$. To define $\boldsymbol{v}(x, y)$, we differentiate between the orthogonal and central projections. We use the visualization in Figure 4 to show this difference. In the case of orthogonal projection, vector $\boldsymbol{v}(x, y)$ defines the $z$-axis (normal vector to the scan plane $\mathcal{P}$ ) and is independent of $x$ and $y$. In the case of central projection, however, vector $\boldsymbol{v}(x, y)$ is defined by the direction of the line connecting the source to the point with coordinates $[x, y, 0]^{\top}$ on $\mathcal{P}$.

Substituting (7) into (10) results in

$$
\begin{aligned}
I(x, y) & =I_{0} e^{-\sum_{n=0}^{N^{3}-1} \mu_{n} \int_{\boldsymbol{v}(x, y)} \psi\left(T^{-1}(\boldsymbol{x})-\boldsymbol{\tau}_{n}\right) \mathrm{d} \lambda} \\
& =I_{0} e^{-\sum_{n=0}^{N^{3}-1} \mu_{n} l_{n}(x, y ; T)},
\end{aligned}
$$




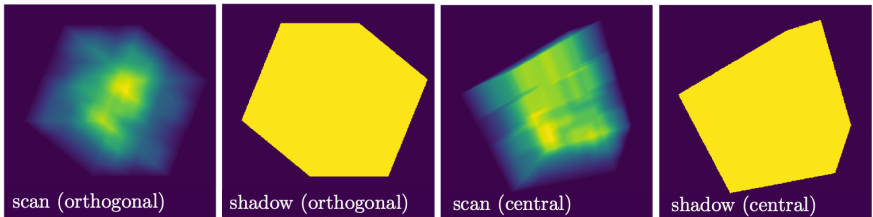

Fig. 5. Examples of scans and shadows for orthogonal and central projections.

where we have defined $l_{n}(x, y ; T)$ as

$$
l_{n}(x, y ; T) \stackrel{\text { def }}{=} \int_{\boldsymbol{v}(x, y)} \psi\left(T^{-1}(\boldsymbol{x})-\boldsymbol{\tau}_{n}\right) \mathrm{d} \lambda .
$$

We choose the notation $l_{n}(x, y ; T)$ to show the dependence of the scan on the orientation of the barcode in space. In other words, $l_{n}(x, y ; T)$ is the length of the intersection segment between a line drawn from point $(x, y)$ in the direction of $\boldsymbol{v}(x, y)$ and the transformed block $\psi\left(T^{-1}(\boldsymbol{x})-\boldsymbol{\tau}_{n}\right)$. This is visualized in Figure 4 for both orthogonal and central projection cases. We can rearrange (11) and define the linearized scan $Q(x, y ; T)$ as

$$
Q(x, y ; T) \stackrel{\text { def }}{=}-\ln \frac{I(x, y)}{I_{0}}=\sum_{n=0}^{N^{3}-1} \mu_{n} l_{n}(x, y ; T) .
$$

If the transformation function $T(\cdot)$ is known, (13) simplifies to a set of linear equations. Suppose that we take $S$ samples of $I(x, y)$ at locations $\left(x_{k}, y_{k}\right)$ with $k \in\{0, \cdots, S-1\}$. Then we have the following matrix notation,

$$
\boldsymbol{q}=\boldsymbol{L}_{T} \boldsymbol{\mu}
$$

where $\boldsymbol{q} \in \mathbb{R}^{S}$ is the vector of linearized sample values transformed using (13) and $\boldsymbol{L}_{T} \in \mathbb{R}^{S \times N^{3}}$ is the matrix of line lengths for rays passing through each cube in the barcode and going through the sampling points in $\mathcal{P}$. Subscript $T$ shows that this matrix depends on the pose of the cube. Finally, $\boldsymbol{\mu} \in \mathbb{R}^{N^{3}}$ is the vector of unknown attenuation coefficients for each cube in the barcode. More precisely,

$$
\boldsymbol{q}[k]=-\ln \frac{I\left(x_{k}, y_{k}\right)}{I_{0}}, \quad \boldsymbol{L}_{T}[k, n]=l_{n}\left(x_{k}, y_{k} ; T\right), \quad \boldsymbol{\mu}[n]=\mu_{n},
$$

for $k \in\{0, \cdots, S-1\}$ and $n \in\left\{0, \cdots, N^{3}-1\right\}$. The line lengths $l_{n}(x, y ; T)$ can be calculated using standard geometric principles (see Appendix A for more details). In practice, the pose function $T(\cdot)$ is not known. However, in the next sections, we provide a way to estimate the unknown pose function $T(\cdot)$. Given this estimate $\widehat{T}(\cdot)$, we can construct matrix $\widehat{\boldsymbol{L}}_{\widehat{T}}$ and compute the attenuation coefficients by inverting the linear system:

$$
\widehat{\boldsymbol{\mu}}=\widehat{\boldsymbol{L}}_{\widehat{T}}^{\dagger} \boldsymbol{q}=\left(\widehat{\boldsymbol{L}}_{\widehat{T}}^{\top} \widehat{\boldsymbol{L}}_{\widehat{T}}\right)^{-1} \widehat{\boldsymbol{L}}_{\widehat{T}}^{\top} \boldsymbol{q} .
$$

The linear formulation in (14) and its inverse in (15) enable algorithms to find the attenuation values from a single scan taken from a known direction.

\section{ESTIMATING THE POSE OF THE BARCODE FROM A SINGLE SCAN}

Reconstructing 3-D objects from tomographic images has been studied extensively [21], [22], [23], [24]. Using a single

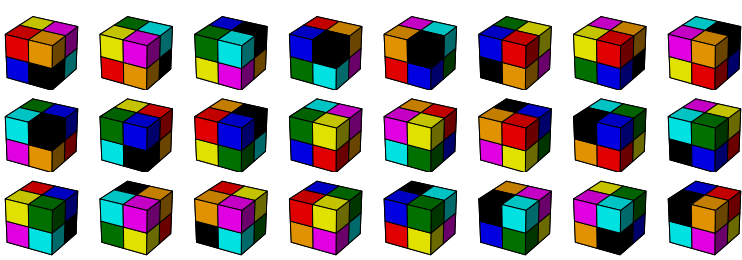

Fig. 6. All 24 possible combinations of code-bit rotations that result in the exact same barcode shadow.

image taken from an unknown direction to reconstruct an object is challenging, if not impossible in the general setting. Fortunately, in our case, we have prior knowledge about the shape of the barcode, which helps in formulating the reconstruction problem mathematically and finding a closedform solution. In order to use this prior information, we need to identify the pose parameters of the barcode, $\omega$ and $t$.

Before going any further, let us provide a definition that is useful throughout the rest of the paper.

Definition 1. The barcode shadow is the convex hull of the eight points formed by the projection of the eight barcode vertices of the cube on $\mathcal{P}$.

The barcode shadows of an example barcode are illustrated in Figure 5 for orthogonal and central projections. One can see how it shows the shape of the scan and it will play an important role in estimating the pose of the barcode. We use some interesting properties of shadows in orthogonal and central projection cases to estimate the pose with high precision. For the orthogonal projection case, the following fact holds:

- The barcode shadow is a square if and only if the image plane is parallel to one of the cube sides, otherwise the barcode shadow is a convex hexagon with three pairs of parallel edges.

For the central projection, the following fact holds:

- The barcode shadow is a convex quadrilateral if the $z$-axis crosses the side of the barcode nearest to $s$. Moreover, if there exists a ray from $s$ to any point on the scan plane which is tangent to a plane constructing a side of the barcode, then the barcode shadow is a convex pentagon. Otherwise, the shadow forms an irregular convex hexagon.

In the following, we show how to use the barcode shadow from a single scan to estimate the pose of the barcode for both orthogonal and central projections. As mentioned in the introduction, this recovery is up to an equivalence class as the barcode shadow is unchanged if the barcode is rotated 90 degrees around any of its axes. Let us look at a simple example in Figure 6 . Here, a $2 \times 2 \times 2$ cubic barcode is rotated in every possible direction. These rotations account for the symmetrical shape of the cubic barcode. The barcode shadow for all these rotations is the same, and thus we will have an ambiguity in the arrangements of the bits in the barcode. For example, the first barcode in the figure with rotation $\left[\omega_{x}, \omega_{y}, \omega_{z}\right]^{\top}$, would produce the same scan and shadow as the second with rotation $\left[\omega_{x}, \omega_{y}-\pi / 2, \omega_{z}\right]^{\top}$. We show in Section $\mathrm{V}$ how to resolve this ambiguity. 


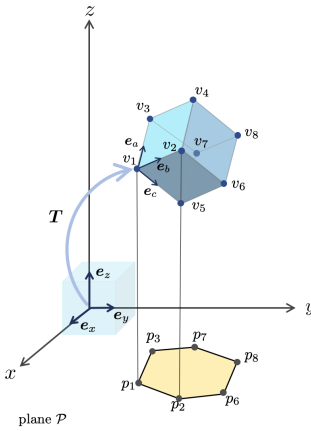

(a)

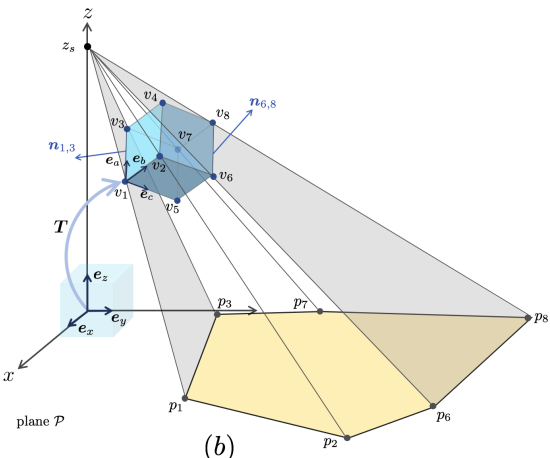

(b)
Fig. 7. Relationship between the barcode orientation and the edges and corners of the produced shadow on the scan plane in the case of (a) orthogonal and (b) central projections.

Figure 7 depicts the notation we will use when deriving the solution to the orientation estimation problem for both orthogonal and central projections. Let us call the vertices of the barcode $v_{i}, i \in\{1, \cdots, 8\}$. Denote the projection of each vertex $v_{i}$ on the scan plane by $p_{i}$. In this section we ignore the special cases (which indeed have a zero measure), and assume that the barcode shadow has six corners. Also define the set of unit vectors $\boldsymbol{e}_{x}, \boldsymbol{e}_{y}$ and $\boldsymbol{e}_{z}$, representing the direction of the edges of the base barcode, as shown in Figure 7. While rotating the barcode in space, these three unit vectors are mapped to the unit vectors $e_{a}, e_{b}$ and $e_{c}$, respectively, according to (9):

$$
\begin{aligned}
& \boldsymbol{e}_{\boldsymbol{a}}=\left[\begin{array}{c}
\cos \omega_{z} \cos \omega_{y} \\
\cos \omega_{y} \sin \omega_{z} \\
-\sin \omega_{y}
\end{array}\right], \\
& \boldsymbol{e}_{\boldsymbol{b}}=\left[\begin{array}{c}
-\sin \omega_{z} \cos \omega_{x}+\sin \omega_{x} \cos \omega_{z} \sin \omega_{y} \\
\cos \omega_{x} \cos \omega_{z}+\sin \omega_{x} \sin \omega_{y} \sin \omega_{z} \\
\sin \omega_{x} \cos \omega_{y}
\end{array}\right], \\
& \boldsymbol{e}_{\boldsymbol{c}}=\left[\begin{array}{c}
\sin \omega_{x} \sin \omega_{z}+\cos \omega_{x} \cos \omega_{z} \sin \omega_{y} \\
-\sin \omega_{x} \cos \omega_{z}+\cos \omega_{x} \sin \omega_{y} \sin \omega_{z} \\
\cos \omega_{x} \cos \omega_{y}
\end{array}\right] .
\end{aligned}
$$

We denote these three vectors as $\boldsymbol{e}_{a}=\left[e_{a, x}, e_{a, y}, e_{a, z}\right]^{\top}, \boldsymbol{e}_{b}=$ $\left[e_{b, x}, e_{b, y}, e_{b, z}\right]^{\top}$, and $\boldsymbol{e}_{c}=\left[e_{c, x}, e_{c, y}, e_{c, z}\right]^{\top}$. From (16) we derive the following closed-form expressions for $\omega_{x}, \omega_{y}$, and $\omega_{z}$ :

$$
\begin{aligned}
\cos \omega_{z} & =\frac{\left|e_{a, x}\right|}{\sqrt{e_{a, x}^{2}+e_{a, y}^{2}}}, \\
\cos \omega_{y} & =\sqrt{e_{a, x}^{2}+e_{a, y}^{2}}, \\
\cos \omega_{x} & =\frac{\left|e_{c, x} \cos \omega_{z} \sin \omega_{y}-e_{b, x} \sin \omega_{z}\right|}{\sin ^{2} \omega_{z}+\cos ^{2} \omega_{z} \sin ^{2} \omega_{y}} .
\end{aligned}
$$

Note that in (17), we assumed that the rotation angles are in the range of $[0, \pi / 2)$. Since we do not know the local coordinate system of the barcode, this orientation ambiguity is inherent and will be later resolved using the Reed-Solomon coding scheme and by considering all the 24 orientation ambiguity cases. According to (17), to find the pose of the barcode in the noiseless case, it is sufficient to find $e_{a, x}, e_{a, y}$, $e_{b, x}$, and $e_{c, x}$.

The general procedure to find the rotation and translation parameters is similar for the orthogonal and central projections, and is as follows:
Step 1: Construct the barcode shadow from the scan.

Step 2: Extract the corners of the barcode shadow.

Step 3: Calculate the elements of $\boldsymbol{e}_{a}, \boldsymbol{e}_{b}$ and $\boldsymbol{e}_{c}$.

Step 4: Given the estimated elements of $\boldsymbol{e}_{a}, \boldsymbol{e}_{b}$ and, $\boldsymbol{e}_{c}$, use (17) to compute $\omega$.

Step 5: Estimate the translation parameters in $\boldsymbol{t}$.

Steps 1 and 2 are the same for both orthogonal and central projections. Steps 3 to 5 however, differ slightly between the two.

In our practical implementation of Step 1, we first denoise the scan by a two-level wavelet decomposition with a Daubechies 2 filter and soft-thresholding with the BayesShrink method [25]. Then, we find the barcode shadow by applying a thresholding followed by a closing morphological transform. In Step 2, we extract the 2-D coordinates of the corners of the barcode shadow using Harris's corner detection method [26].

In Steps 3 to 5, we leverage the geometrical properties of our barcode to find $\boldsymbol{\omega}$ and $\boldsymbol{t}$. We explain these steps separately for each projection type below.

\section{A. Orthogonal Projection}

It is easy to see in Figure $7 \mathrm{a}$ that

$$
\left[\boldsymbol{p}_{3}-\boldsymbol{p}_{1}, \boldsymbol{p}_{2}-\boldsymbol{p}_{1}, \boldsymbol{p}_{6}-\boldsymbol{p}_{2}\right]=N d\left[\begin{array}{ccc}
1 & 0 & 0 \\
0 & 1 & 0 \\
0 & 0 & 0
\end{array}\right]\left[\boldsymbol{e}_{a}, \boldsymbol{e}_{b}, \boldsymbol{e}_{c}\right],
$$

where $\boldsymbol{p}_{i}$ is the 3-D coordinate of the shadow corner $p_{i}$, and $N d$ is the length of each edge of the cube. We can derive $e_{a, x}$, $e_{a, y}, e_{b, x}$, and $e_{c, x}$ from (18) and substitute them into (17) to find closed-form solutions for $\omega_{x}, \omega_{y}$, and $\omega_{z}$. With estimated $\omega$, we can find $e_{a}, e_{b}$, and $e_{c}$ from (16).

In the next step, to find the translation parameters in $t$, we connect all the corners of the shadow to their next third corner. The three lines will intersect in a single point - call it $\bar{p}-$, which is the projection of the cube center on the scan plane. We can then write

$$
\boldsymbol{t}=\overline{\boldsymbol{p}}-\frac{N d}{2}\left(\boldsymbol{e}_{a}+\boldsymbol{e}_{b}+\boldsymbol{e}_{c}\right)+\left[\begin{array}{l}
0 \\
0 \\
\zeta
\end{array}\right]
$$

Note that while performing an orthogonal projection, the information about the barcode translation in the $z$-axis is lost and the free parameter $\zeta$ represents this. Also, note that the association of the shadow edges to the $e_{a}, e_{b}$, and $e_{c}$ can be different than our choice in $(18)$ as long as the three vectors satisfy the right-hand rule. This ambiguity about the rotation of the barcode is part of what we show in Figure 6 and will be resolved later.

\section{B. Central Projection}

We use the notation in Figure $7 \mathrm{p}$ to visualize the derivation of the barcode pose from its shadow corners in the central projection case. Consider the plane $\mathcal{P}_{1,3}$, with normal $\boldsymbol{n}_{1,3}$ in Figure 7b, defined as the plane passing through $p_{1}, p_{3}$ and the source $s$. Plane $\mathcal{P}_{1,3}$ contains the edge $v_{1}-v_{3}$ from the cube, thus $\boldsymbol{n}_{1,3}$ is orthogonal to $v_{1}-v_{3}$. Similarly, plane $\mathcal{P}_{6,8}$, with normal $\boldsymbol{n}_{6,8}$, contains the edge $v_{6}-v_{8}$. Since the edges $v_{1}-v_{3}$ and $v_{6}-v_{8}$ are parallel to each other, the normal vectors $\boldsymbol{n}_{1,3}$ 


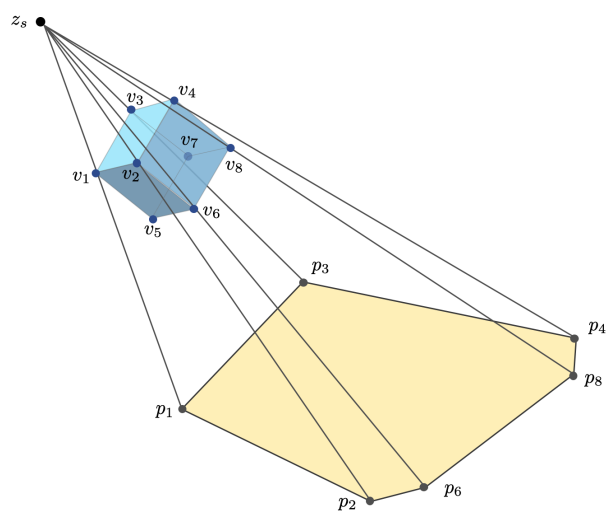

Fig. 8. Special class of cases, where opposite edges of the barcode shadow do not correspond to parallel barcode edges.

and $\boldsymbol{n}_{6,8}$ are orthogonal to both these lines. Hence, their cross product produces the direction of these edges, represented by the unit vector $e_{a}$ in the figure. By applying the same procedure on the other corners of the barcode shadow we can also identify unit vectors $\boldsymbol{e}_{b}$ and $\boldsymbol{e}_{c}$ as

$$
\begin{gathered}
\boldsymbol{e}_{a}=\frac{\boldsymbol{n}_{1,3} \times \boldsymbol{n}_{6,8}}{\left\|\boldsymbol{n}_{1,3} \times \boldsymbol{n}_{6,8}\right\|}, \quad \boldsymbol{e}_{b}=\frac{\boldsymbol{n}_{7,8} \times \boldsymbol{n}_{1,2}}{\left\|\boldsymbol{n}_{7,8} \times \boldsymbol{n}_{1,2}\right\|}, \\
\boldsymbol{e}_{c}=\frac{\boldsymbol{n}_{3,7} \times \boldsymbol{n}_{2,6}}{\left\|\boldsymbol{n}_{3,7} \times \boldsymbol{n}_{2,6}\right\|} .
\end{gathered}
$$

Now that we have calculated $e_{a}, e_{b}$ and $e_{c}$, we can use (17) to find a closed form solution for $\boldsymbol{\omega}$.

Next, to find the translation parameters in $t$, we first note that each projected vertex of the cube $v_{i}$, can be represented as

$$
\boldsymbol{v}_{i}=\left[\begin{array}{c}
\alpha_{i} x_{i} \\
\alpha_{i} y_{i} \\
\left(1-\alpha_{i}\right) z_{s}
\end{array}\right] .
$$

where $\left[x_{i}, y_{i}\right]^{\top}$ is the coordinate of the shadow corner $p_{i}$ on the scan plane $\mathcal{P}$, and $\boldsymbol{v}_{i}$ contains the coordinates of $v_{i}$. Also note from Figure $7 \mathrm{p}$ that $\boldsymbol{v}_{1}+N d \boldsymbol{e}_{a}=\boldsymbol{v}_{3}$. We can write the same relationship for all the adjacent corners of the barcode shadow and solve a set of linear equations to find the values of $\alpha_{i}$, and thus the values of $\boldsymbol{v}_{i}, i \in\{1,2,3,6,7,8\}$. The barcode cube center - call it $\overline{\boldsymbol{v}}$ - is the average of the identified cube vertices. With this, we can find the translation vector $t$ as

$$
\boldsymbol{t}=\overline{\boldsymbol{v}}-\frac{N d}{2}\left(\boldsymbol{e}_{a}+\boldsymbol{e}_{b}+\boldsymbol{e}_{c}\right)
$$

In the example in Figure 7p for central projection, we assumed that opposite edges of the barcode shadow (each edge and the next third one) correspond to parallel edges of the barcode cube (e.g. $p_{1}-p_{3}$ and its third next neighbor, $p_{6}-p_{8}$ correspond to two parallel barcode edges $v_{1}-v_{3}$ and $v_{6}-v_{8}$ ). For a small set of arrangements of the barcode and the source, this assumption is not true. We see an example in Figure 8 , where the opposite shadow edges $p_{2}-p_{6}$ and $p_{3}-p_{4}$ do not correspond to parallel edges of the barcode cube. We can identify such cases by simply connecting the opposite shadow corners and seeing that they do not intersect in a single point. In this case, out of the three pairs of opposite shadow edges, only one pair corresponds to parallel edges of the cube, for example, the pair $p_{1}-p_{3}$ and $p_{6}-p_{8}$ in Figure 8. Thus, we need to consider $\mathcal{P}_{1,3}$ with $\mathcal{P}_{6,8}, \mathcal{P}_{1,2}$ with $\mathcal{P}_{3,4}$, and $\mathcal{P}_{2,6}$ with $\mathcal{P}_{4,8}$ to estimate the barcode pose. It is easy to show that, almost surely, only this choice of pairs of planes results in $\boldsymbol{e}_{a}$, $\boldsymbol{e}_{b}$ and $\boldsymbol{e}_{c}$ being orthogonal (more details are provided in the supporting material). Hence, if we fall under this special case, we test the three pairs of opposite shadow edges, and keep the one that results in an orthogonal set of $e_{a}, e_{b}$, and $e_{c}$.

\section{Fine-tuning}

Although straightforward, the geometrical approach that we presented above does not directly take into account that the barcode shadow is generated from a cube. Leveraging this additional information can be beneficial in the presence of noise. In order to enforce this condition on the barcode, we apply further fine-tuning on the estimated pose parameters using a gradient descent optimizer. To this end, we use a numerical non-linear optimization method to minimize the cost function defined as

$$
f(\boldsymbol{w}, \boldsymbol{t})=\|\boldsymbol{p}(\boldsymbol{w}, \boldsymbol{t})-\widetilde{\boldsymbol{p}}\|_{F}^{2},
$$

where $\boldsymbol{p}(\boldsymbol{w}, \boldsymbol{t}) \in \mathbb{R}^{6 \times 2}$ is the coordinates of the corners of the shadow of a barcode rotated and translated by $\boldsymbol{w}$ and $t$, and $\widetilde{\boldsymbol{p}}$ is the observed coordinates of the barcode shadow. We use the output of the geometrical approach as the initial solution for the non-linear optimizer.

In Figure 9, we provide an example of estimating the orientation using the above procedure for central projection. Here, the ground truth cube has $\boldsymbol{\omega}=\pi / 180 \times[50,15,70]^{\top}$ and $t=[1.5,-4,33]^{\top}$. The corners of its scan are shown by black dots in Figure 9 $\mathrm{b}$. We then perturb these corners by a uniform noise in $[-0.1,0.1]^{2}$. The perturbed corners are shown by the gray squares in Figure $9 \mathrm{p}$. Then, we estimate the pose parameters using only the geometrical approach, only Gradient Descent (GD) with an initial solution set to $\left(\boldsymbol{\omega}_{0}, \boldsymbol{t}_{0}\right)=(\mathbf{0}, \mathbf{0})$, and with the combination of the two (geometrical + GD). In the legend we report the relative reconstruction error, defined as

$$
\left[\frac{\|\omega-\widehat{\omega}\|}{\|\omega\|}, \frac{\|t-\widehat{t}\|}{\|t\|}\right] .
$$

In the reconstruction, we can see that the GD method gets stuck in a local minimum, far from the ground truth. The geometrical approach finds a better result, but when followed by a gradient descent fine-tuning, the reconstruction improves significantly, leading to relative errors equal to [0.010, 0.005].

\section{Unique Reconstruction of the Code}

Once we extract the pose parameters using the procedures explained in Sections III-A to III-C, we can calculate $l_{n}(x, y ; T)$, which enables us to invert the linear equation in (14) and estimate the values of attenuation coefficients, $\mu_{n}$. We can prove that with this procedure, the embedded code bits in the barcode can be recovered uniquely up to a known set of 24 permutations shown in Figure 6

Lemma 1. The pose reconstruction algorithm in Steps 1 to 5 and explained in Sections III-A and III-B, followed by solving 


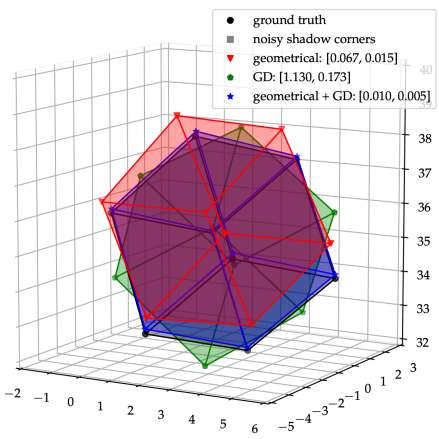

(a)

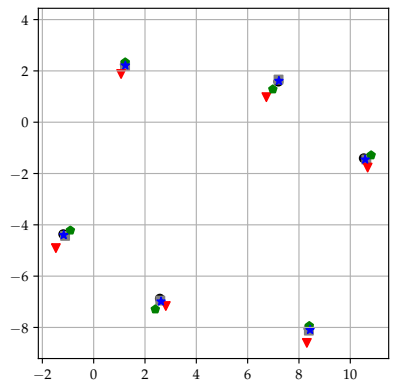

(b)
Fig. 9. (a) A cubic barcode (in black) and its reconstructions using different methods; (b) corners of the scan of the cubic barcode (black), perturbed corners with noise (gray), and corners of the scans of reconstructions of the barcode based on estimated pose parameters $\widehat{\omega}$ and $\widehat{t}$. Numbers in the legend correspond to the relative reconstruction errors defined in 21 .

the inverse problem in (15) recovers the embedded barcode bits uniquely up to a set of 24 permutations shown in Figure 6 . provided that we have enough samples from the scan such that the matrix $\boldsymbol{L}_{T}$ has full rank.

Proof. We provide the proof in Appendix B

\section{SAMPLing TheOREM FOR ORTHOgONAL PROJECTION}

In the reconstruction process, since we have the whole scan, we can use all the pixel values for better robustness against noise. However, it is interesting to know the minimum number of samples required in the noiseless scenario to guarantee a reconstruction in theory. The minimum number of samples defines the maximum pixel size in the scan image and thus the minimum reconstructable scan resolution. Since the scan values for the central projection case depend not only on $\boldsymbol{\omega}$ and $\boldsymbol{t}$, but also on $z_{s}$, finding the answer to this question becomes very complicated. Here we focus on the case of orthogonal projection to answer this question.

We define a bit region for each particular bit voxel as the region over the scan plane in which the projection of that bit voxel lies. This region plays an important role in the sampling process during reconstruction, as all the information about one bit value exclusively appears inside its bit region. Without a sample from this region, the information about that bit is completely lost. The following lemma states a bound for the maximum distance between samples that guarantees at least one measurement from each bit region.

Lemma 2. Consider a cubic barcode with $N^{3}$ bits carried over voxels of side length $d$. The barcode is scanned by a parallel beam scanner over plane $\mathcal{P}$ in an arbitrary direction $\boldsymbol{v}(x, y)$. A uniform sampling inside the barcode shadow, with spacing $D_{x}$ and $D_{y}$ along the $x$ and $y$ axes guarantees at least one sample from each code-bit region over $\mathcal{P}$ if $D_{x}, D_{y} \leq d \sqrt{2} r_{c}\left(\omega_{x}, \omega_{y}\right)$, where $r_{c}\left(\omega_{x}, \omega_{y}\right)$ is the radius of the inscribed circle inside a bit region, illustrated in Figure 10p.

Proof. The proof of this lemma is given in Appendix C.

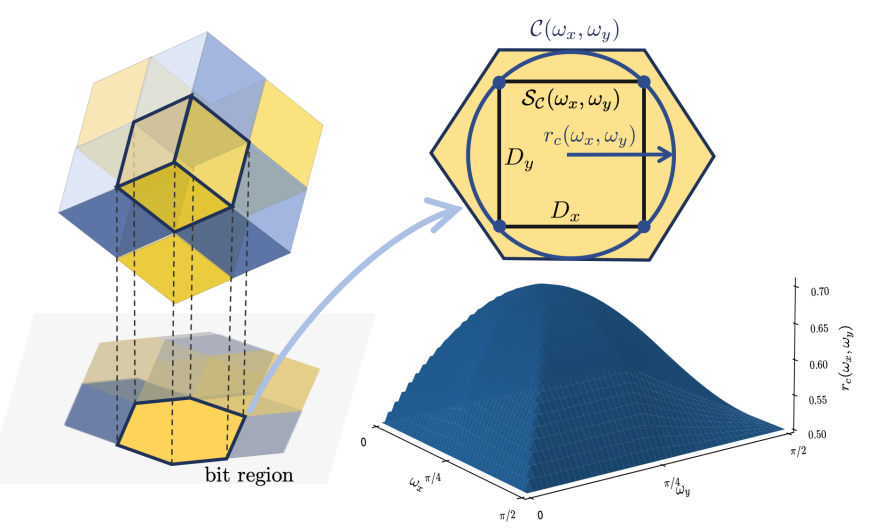

Fig. 10. (a) Definition of the bit region and sampling with at least one sample at each bit region. (b) Values of the radius of inscribed circle $r_{c}\left(\omega_{x}, \omega_{y}\right)$.

Lemma 2 shows the relation between the number of samples and the size of the voxels carrying the barcode information, as well as the scanning direction. We can see that the smaller the voxel size length, $d$ is, the smaller the sampling period needs to be. Moreover, as we have seen before, for some special cases, e.g. when $\omega_{x}=\omega_{y}=0$, no reconstruction algorithm can find the bit values using a single scan. In the above lemma, we do not consider the difference between the attenuation coefficients of the materials that are used to construct the barcode. Theoretically, if the two materials are different, we can reconstruct the barcode bits. In the presence of noise, however, we need to have materials with significantly different attenuation coefficients to be able to reconstruct the barcode information. We leave finding the relationship between the material attenuation coefficients and the sampling requirements as future work.

\section{CODING SCHEMES FOR CUBIC BARCODES}

It is common to use error correction codes in noisy communication channels to retrieve the correct information bits despite some toggled bit values. Normally, in an error correction code, a partial redundancy is added to the codebits to guarantee detection of erroneous bits and correct reconstruction up to some noise level. In our cubic barcode structure, we use channel coding in order to achieve two advantages:

1) Correct errors generated during the scanning and/or reconstructing the barcode

2) Resolve the ambiguity around the barcode orientation.

Recall from Section III that we can find the barcode orientation using the barcode shadow up to an equivalence class, where the bits can form one of possible 24 arrangements. These arrangements result in structured permutations in the code-bits when reconstructed. Only one of such permutations has the correct correspondence of the information bits to the added redundant parity bits in the coded message. Provided that the number of erroneous bits caused by the sampling and reconstruction process does not surpass the error correction capability of the code, that correct bit arrangement will pass the decoder.

To encode the barcode information, we choose ReedSolomon (RS) codes. RS codes are linear error-correcting 


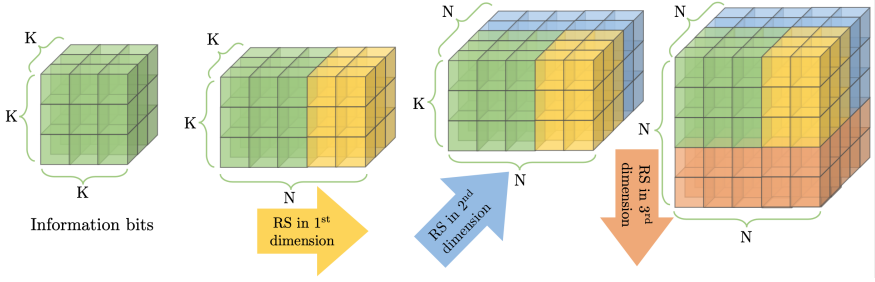

Fig. 11. Interpretation of 3-D Reed-Solomon coding scheme in terms of 1-D conventional Reed-Solomon.

codes that Reed and Solomon introduced in 1960 [27]. They are widely used in CDs [28], DVDs [29], QR codes [30], data transmission technologies such as DSL [31], and broadcast systems such as DVB [32].

We start by providing a quick recap of one-dimension Reed-Solomon codes adapted to our cubic barcode structure. Consider the finite field $\mathbb{Z}_{q}$ as the set of integers modulo $q$, with $q$ a prime number. When $q$ is finite, we call $\mathbb{Z}_{q}$ a Galois field and represented it as $G F(q)$. An element $\alpha \in \mathbb{Z}_{q}$ is called a primitive element of $G F(q)$, if $\alpha^{q-1}=1$. Every element of $G F(q)$ can be written as a power of $\alpha$. Thus, the set $\left\{1, \alpha, \alpha^{2}, \cdots, \alpha^{q-2}\right\}$ is a basis for $G F(q)$. Reed-Solomon codes are constructed by utilizing these basis vectors. A ReedSolomon code of length $N_{O}=q-1$ and dimension $K_{I} \leq N_{O}$ over a field $\mathbb{Z}_{q}$ is constructed from the set of all degree $K_{I}-1$ polynomials

$$
R(x)=\sum_{k=0}^{K_{I}-1} s_{i} x^{k}
$$

where $s_{k}, k \in\left\{0, \cdots, K_{I}-1\right\}$ are the input symbols (to be encoded) and the elements of the set

$$
\left\{\left(r_{0}, r_{1}, \cdots, r_{N_{O}-1}\right) \mid r_{i}=R\left(\alpha^{i}\right), i=0,1, \cdots, N_{O}-1\right\},
$$

are the output symbols. The resulting RS code, represented by $R S\left(N_{O}, K_{I}\right)$, can correct up to $0.5\left(N_{O}-K_{I}\right)$ erroneous symbols. We review a different representation of Reed-Solomon codes using generalized Fourier transforms in the supporting material document, that can help in using them in different applications, including orientation disambiguation of cubic barcodes.

\section{A. 1-D Reed-Solomon}

In the first scheme, we assume that we want to generate $N^{3}$ Reed-Solomon code bits from $K$ information bits. If we define each information byte as a symbol, then $N_{O}=N^{3} / 8$ and $K_{I}=K / 8$. We construct $R S\left(N_{O}, K_{I}\right)$ that results in $N^{3}$ code bits from $K_{I}$ input bytes. We rearrange these $N^{3}$ bits into a 3-D tensor as our barcode. Thus, we encode $K$ information bits into $N^{3}$ RS bits in our cubic barcode. Here we assumed that $N^{3}$ and $K$ are multiples of 8 . This is a design factor that we can take into account while building the barcode.

The error correction capability (ECC) of this scheme in terms of the number of bytes is

$$
E C C=\frac{N_{O}-K_{I}}{2}=\frac{N^{3}-K}{8 \times 2} .
$$

\section{B. 3-D Reed-Solomon}

Suppose that we have $K_{1}^{3}$ information bits, arranged in a $K_{1} \times K_{1} \times K_{1}$ tensor. We first group them into $2 \times 2 \times 2$ blocks each of which we call a symbol. We thus have a $K_{1} / 2 \times K_{1} / 2 \times K_{1} / 2$ tensor of symbols, which we will use to construct a 3-D Reed-Solomon code. Let us define $K_{I}=K_{1} / 2$ and $N_{O}=N / 2$. With these definitions, the input sequence is a $K_{I} \times K_{I} \times K_{I}$ structure and the output is a $N_{O} \times N_{O} \times N_{O}$ structure whose symbols are numbers in $\{0,1, \cdots, 255\}$; here, each symbol has 256 possible values since it is built from $2 \times 2 \times 2=8$ bits. We generate the 3 -D Reed-Solomon output symbols from input symbols as follows:

$$
r_{l, m, n}=\sum_{i, j, k=0}^{K_{I}-1} s_{i, j, k} \alpha^{i l+j m+k n}, \quad l, m, n \in\left\{0, \cdots, N_{O}-1\right\} .
$$

Since the summation in the above equation is separable, generating the 3-dimensional RS code $r_{l, m, n}$ is equivalent to applying the one-dimensional transformations consecutively to each dimension of the input tensor. The 3-D RS scheme is interpreted in terms of the conventional 1-D RS coding scheme in Figure 11.

The error correction capability of the proposed 3-D RS code is derived as follows,

$$
\begin{aligned}
E C C & =K_{I}^{2} \frac{N_{O}-K_{I}}{2}+K_{I} N_{O} \frac{N_{O}-K_{I}}{2}+N_{O}^{2} \frac{N_{O}-K_{I}}{2} \\
& =\frac{N_{O}-K_{I}}{2}\left(K_{I}^{2}+K_{I} N_{O}+N_{O}^{2}\right)=\frac{N^{3}-K_{1}^{3}}{8 \times 2} .
\end{aligned}
$$

\section{1-D vs 3-D Reed-Solomon}

By comparing (22) and 23), we see that for $K=K_{1}^{3}$ the two schemes have the same error correction capability. However, there are other factors that contribute to the choice of the coding scheme. For example, when $N \leq 8$, since the number of bytes in each dimension is too small, it is not possible to generate RS codes with error correction for each dimension separately and it is preferable to aggregate the code bytes and generate a 1D RS code for the whole sequence. Also when $K$ is not a cube number, we are bound to using 1-D RS codes, since 3-D codes work only when $K$ is a cube number. On the other hand, 3-D Reed-Solomon scheme works better for large values of $N$, as it computes the code in each dimension consecutively, while it is faster to generate RS codes with length $N$ than length $N^{3}$.

\section{Ambiguity Resolution}

In this section, we delegated the task of resolving the barcode orientation ambiguity to the coding scheme. Assuming that the scanning and reconstructing procedures have not introduced too many errors in the code-bits, the correct bit arrangement (out of the 24 possible arrangements) will pass the decoder test. Through all the tests that we ran with this method, we did not run into a case, where more than one of those 24 arrangements passed the decoder test. However, we have not been able to prove this result theoretically, and leave it as a conjecture to be proved in a future work. 
Alternatively, one can designate certain code bits in the barcode to identify the barcode's local coordinate system, similar to the currently implemented 2-D QR barcodes. Although we believe in the potential of this approach, we do not explore it in this article.

\section{THE ROBUSTNESS ANALYSIS OF BARCODE ORIENTATION}

Recall from (14) that the scanning framework in a cubic barcode can be modeled as a linear system of equations. In this section, we analyse the robustness of the process to different barcode orientations, by upper bounding the amount of information that is recoverable from a projection at a particular angle. We can already have an intuitive guess on the relation of the amount of recoverable information in the scan and the orientation. For example, in the case of orthogonal projection, when the scan plane $\mathcal{P}$ is parallel to a side of the cube, some of the bit regions lie on top of each other and appear on the same region on the scan plane. Therefore, we expect that for the cases with this type of symmetricity, the amount of information present in the projection is less than the case where the barcode is scanned in a non-symmetric direction such as $\omega_{x}=30^{\circ}$ and $\omega_{y}=50^{\circ}$. In the former case, changing the order of the rows of the bits in the cube, does not change the scan value, while in the latter, the orientation is such that we receive information from all the bits in the scan. In this section, the goal is not to have a capacity analysis for the barcode, instead, we are interested in finding out how robust is each orientation in terms of reconstructing the bit values from the scan image. As long as $\boldsymbol{\mu}$ is retrieved from the scan, the bit values are not important in this analysis, therefore, we assume a general case in which the bit values are arbitrary and not just taken from a binary set, i.e. $\boldsymbol{\mu} \in \mathbb{R}^{N^{3}}$. We further assume that the scan samples are contaminated by some additive i.i.d. random Gaussian noise $\boldsymbol{\nu} \sim \mathcal{N}\left(0, \sigma_{\nu}^{2} I_{S}\right)$, independent of the attenuation coefficients $\boldsymbol{\mu}$.

The sampling process can be formulated as follows,

$$
\boldsymbol{q}=\boldsymbol{L}_{T} \boldsymbol{\mu}+\boldsymbol{\nu},
$$

where $\boldsymbol{\mu} \in \mathbb{R}^{N^{3}}, \boldsymbol{q} \in \mathbb{R}^{S}$ and $\boldsymbol{L}_{T} \in \mathbb{R}_{+}^{S \times N^{3}}$. The formulation in (24) mimics a communication channel. We can compute the recoverable information of the barcode as follows:

$$
\begin{aligned}
\mathcal{I}(\boldsymbol{\mu} ; \boldsymbol{q}) & =h(\boldsymbol{q})-h(\boldsymbol{q} \mid \boldsymbol{\mu})=h(\boldsymbol{q})-h\left(\boldsymbol{L}_{T} \boldsymbol{\mu}+\boldsymbol{\nu} \mid \boldsymbol{\mu}\right) \\
& =h(\boldsymbol{q})-h(\boldsymbol{\nu}),
\end{aligned}
$$

where $\mathcal{I}(\boldsymbol{\mu} ; \boldsymbol{q})$ is the mutual information between $\boldsymbol{\mu}$ and $\boldsymbol{q}$, assuming that matrix $\boldsymbol{L}_{T}$ is known, and $h(\cdot)$ is the entropy function.

We assume an uncorrelated Gaussian noise with covariance matrix $\boldsymbol{\Lambda}_{\nu}=\sigma_{\nu}^{2} I_{S}$ and define $\boldsymbol{\Lambda}_{\boldsymbol{\mu}}=\mathbb{E}\left(\boldsymbol{\mu} \boldsymbol{\mu}^{T}\right)$ as the covariance matrix of input sequence $\boldsymbol{\mu}$. If code-bits were generated from binary set $\mu_{i, j, k} \in\left\{\mu_{\text {low }}, \mu_{\text {high }}\right\}$ with uniform distribution, then the average power for each code-bit was $P_{T}=\left(\mu_{\text {low }}^{2}+\mu_{\text {high }}^{2}\right) / 2$.

In order to evaluate the maximum carried information, we consider input code-bits to have Gaussian distribution with the same average code-bit power. In other words, $\boldsymbol{\Lambda}_{\mu}=$
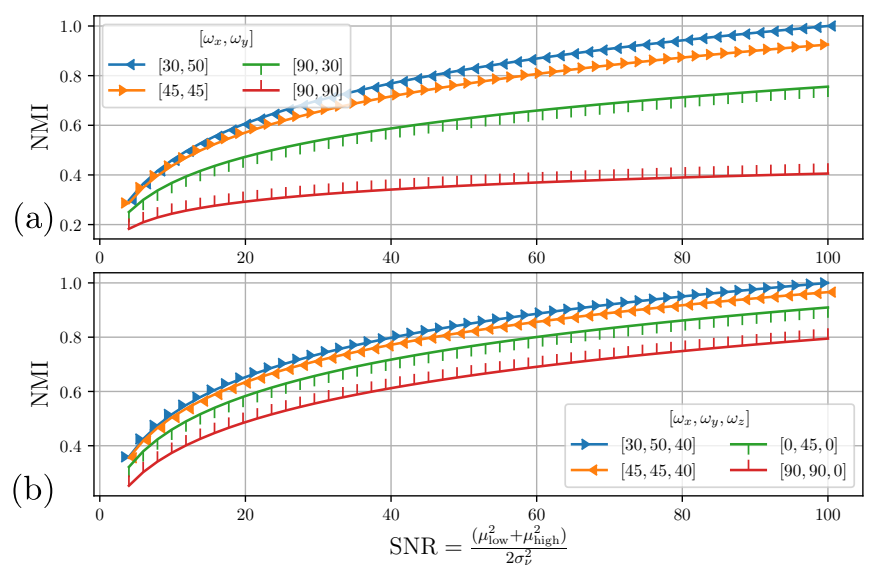

Fig. 12. Normalized maximum mutual information that can be carried over the scan of cubic barcode, with $N=4$ for (a) orthogonal and (b) central projections with some selected barcode orientations.

$P_{T} I_{N^{3}} / N^{3}$. We can verify that the mutual information is maximized for Gaussian distribution of $\boldsymbol{q}$ and is derived as

$$
\begin{aligned}
\mathcal{I}(\boldsymbol{\mu} ; \boldsymbol{q}) & \leq \log _{2}\left((2 \pi e)^{S} \operatorname{det}\left(\boldsymbol{L}_{T} \boldsymbol{\Lambda}_{\boldsymbol{\mu}} \boldsymbol{L}_{T}^{T}+\boldsymbol{\Lambda}_{\boldsymbol{\nu}}\right)\right)-\log _{2}\left((2 \pi e)^{S} \operatorname{det}\left(\boldsymbol{\Lambda}_{\boldsymbol{\nu}}\right)\right) \\
& =\log _{2}\left(\operatorname{det}\left(I_{S}+\boldsymbol{L}_{T} \boldsymbol{\Lambda}_{\boldsymbol{\mu}} \boldsymbol{L}_{T}^{T} \boldsymbol{\Lambda}_{\boldsymbol{\nu}}^{-1}\right)\right) \\
& \stackrel{(a)}{=} \log _{2}\left(\operatorname{det}\left(I_{S}+\frac{P_{T}}{N^{3} \sigma_{\boldsymbol{\nu}}^{2}} \boldsymbol{U}_{\boldsymbol{L}_{T}} \boldsymbol{\Lambda}_{\boldsymbol{L}_{T}} \boldsymbol{U}_{\boldsymbol{L}_{T}}^{T}\right)\right) \\
& \stackrel{(b)}{=} \sum_{n=1}^{N^{3}} \log _{2}\left(1+\frac{P_{T}}{N^{3} \sigma_{\boldsymbol{\nu}}^{2}} \lambda_{\boldsymbol{L}_{T}}^{(n)}\right)
\end{aligned}
$$

Here, in $(a)$ we have replaced $\boldsymbol{L}_{T} \boldsymbol{L}_{T}^{\top}$ with its eigenvalue decomposition $\boldsymbol{L}_{T} \boldsymbol{L}_{T}^{\top}=\boldsymbol{U}_{\boldsymbol{L}_{T}} \boldsymbol{\Lambda}_{\boldsymbol{L}_{T}} \boldsymbol{U}_{\boldsymbol{L}_{T}}^{\top}$ and in (b), we have defined $\lambda_{\boldsymbol{L}_{T}}^{(n)}$ as the $n$-th non-zero eigenvalue of $\boldsymbol{L}_{T} \boldsymbol{L}_{T}^{\top}$.

In Figure 12, we show the Normalized maximum Mutual Information (NMI) of the barcode scanned from different directions vs the SNR for $N=4$; here, SNR is defined as $\mathrm{SNR}=\left(\mu_{\text {low }}^{2}+\mu_{\text {high }}^{2}\right) /\left(2 \sigma_{\nu}^{2}\right)$. The normalization is done in order to have a better scale on the figures for comparison. For normalization, the maximum information calculated in 26 is divided by the maximum value, which has been seen in the simulation in the orthogonal and central projection cases. Recall that the value of $\omega_{z}$ does not change the scan values in the orthogonal projection case. Thus, in the experiment for orthogonal projection, we set it to zero and do not change it. We can see that $\left(\omega_{x}, \omega_{y}\right)=(30,50)$ in the orthogonal projection case and $\left(\omega_{x}, \omega_{y}, \omega_{z}\right)=(30,50,40)$ for central projection, provide higher recoverable information than several others that would cause ambiguity in the reconstructed code bits, such as $\left(\omega_{x}, \omega_{y}\right)=(90,90)$ in orthogonal projection case and $\left(\omega_{x}, \omega_{y}, \omega_{z}\right)=(90,90,0)$ in central projection case.

\section{Simulation AND Real EXPERIMENT}

In order to assess the performance of our proposed barcode reconstruction algorithms, we test them both with simulations and a real experiment. We embed the same information bits in the barcode in all the simulations as well as the real experiment. We explain the details of this barcode design in Section VII-A In a first test, we investigate the stability of our reconstruction algorithm versus noise and barcode pose 


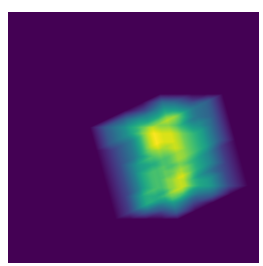

(a)

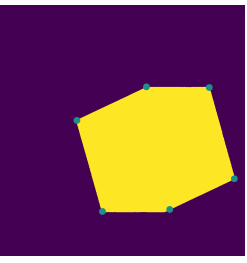

(b)

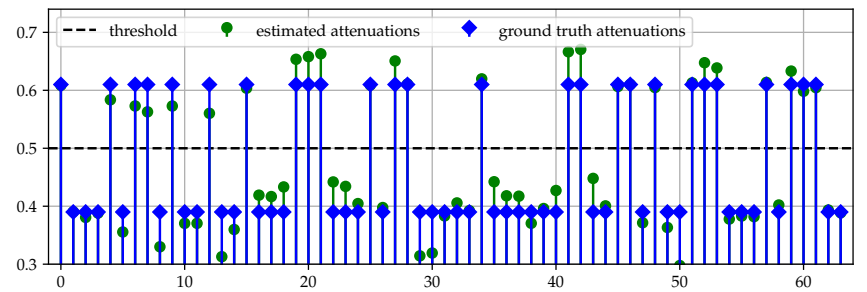

(d)

Fig. 13. Reconstruction of code bit values from simulation of the scan from orthogonal projection. (a) simulation of noisy scan, (b) barcode shadow along with the extracted corners using Harris's corner detection method, (c) comparison of reconstructed corners from orientation parameters calculated by geometrical and gradient descent methods, (d) reconstructed attenuation values compared to the ground truth.

for both orthogonal and central projections. Then, through a simulation, we provide an example of the procedure to reconstruction the information bits from an orthogonal projection of the barcode. Finally, in the case of central projection, we showcase a real implementation of the aforementioned cubic barcode and provide the reconstruction framework from a scan taken by a cone-beam X-ray scanner (the most common type of industrial scanner).

\section{A. Barcode Design and Implementation}

We designed a cubic barcode with $N=4$. Thus, the barcode has in total 64 code-bits. We reserved 48 bits as information bits, and applied an $R S(8,6)$ one-dimensional Reed-Solomon coding scheme on them, as in Section V-A. Since we have two check symbols (16 bits), our implementation has the ability to correct one erroneous symbol (byte), i.e. 8 consecutive bits. Therefore, we can only guarantee to correct one erroneous bit, since two erroneous bits in different symbols cannot be corrected; however, in principle, a maximum of eight erroneous bits can be corrected if they are all in the same symbol. We also assume that $d=1$, resulting in a $4 \mathrm{~cm} \times 4 \mathrm{~cm} \times 4 \mathrm{~cm}$ barcode. In Figure 17a, you can see the 3-D barcode, where black cubes correspond to 1 and yellow cubes to 0 .

The barcode is constructed with materials with attenuation coefficients of 0.61 and 0.39 to represent zeros and ones, respectively ${ }^{11}$ In order to make the simulation and real experiment similar, we choose the same attenuation coefficients for simulations.

\footnotetext{
${ }^{1}$ Since higher attenuation results in a weaker signal on the scan plane, we represent zeros by material with a larger attenuation coefficient. We explain this specific choice of values in Section VII-E
}

\section{B. Orthogonal Projection Simulation}

We assume that the barcode, with the design in Section VII-A is scanned using orthogonal projection.

The pose parameters of the barcode are set to $\boldsymbol{\omega}=$ $\left[70^{\circ}, 50^{\circ}, 0^{\circ}\right]$ and $\boldsymbol{t}=[0,0,0]$ (recall that in the case of orthogonal projection, $\omega_{z}$ and $\boldsymbol{t}$ do not affect the shape of the scan. Thus, we set them to zero in this experiment). We assume that the scan plane is located on the $x y$-plane. In order to make the simulation more realistic, we assume that the scan is perturbed with some small white Gaussian noise such that we have a PSNR $=57 \mathrm{~dB}$. Here, we add the noise to make the problem of extracting the parameters non-trivial. The noisy scan is shown in Figure 13a

We first construct the barcode shadow according to Step 1 in Section III Then we follow with Step 2 and using Harris's corner detection method, find the six corners of the barcode shadow. This is depicted in Figure 13b. In Steps 3 to 5 . we follow the procedure in Section III-A to estimate the parameters of the barcode pose. In Figure 13c we show the barcode shadow corners simulated using the estimated parameters by only the geometrical approach as well as the combination with the fine-tuning, compared to the extracted corners from the barcode shadow in the previous step. We can see that the fine-tuning step is helpful in estimating the barcode pose parameters. Once the values for $\boldsymbol{\omega}$ and $\boldsymbol{t}$ (equivalently the transformation $T$ ) are estimated, we can use the linear inverse equation in (15) to find the attenuation coefficients $\mu_{n}$. Next, we use a threshold of 0.5 (the middle point of 0.39 and 0.61 ) to find the bits. We decode all the values above this threshold as 0 and all below as 1 . Finally, we construct all the 24 arrangements of these bits according to Figure 6 and choose the one that passes the decoder test. The values of the reconstructed $\mu_{n}$ for the correct estimated orientation are shown in Figure 13d, along with the true ground-truth attenuation coefficients.

\section{Success Rate vs. Noise and Barcode Orientation}

We aim at testing the stability of our reconstruction algorithm to noise as well as to the barcode pose in space. For example, we have seen that in the case that $\boldsymbol{\omega}=\mathbf{0}$ for orthogonal projection, there is no way to successfully reconstruct the barcode from a single projection. For each case, we compute the matrix $\boldsymbol{L}_{T}$ and $\boldsymbol{q}$ as in (14), solve (15), threshold the estimated attenuation coefficients to find the bit values, and finally, use Reed-Solomon decoding to extract and correct the information bits.

Here, we are interested in assessing the robustness of the inverse problem in (15) to the pose parameters, and not the pose estimation approach. Thus, in this experiment, we assume that the pose parameters $\boldsymbol{\omega}$ and $\boldsymbol{t}$ are known to the reconstruction algorithm and skip the pose estimation step.

In order to add noise to the projections, we add a zeromean white Gaussian noise $\nu \sim \mathcal{N}\left(0, \sigma_{\nu}^{2}\right)$, with given $\sigma_{\nu}$, and construct the noisy scan readings by

$$
\widetilde{Q}(x, y ; T)=\max (Q(x, y ; T)+\nu, 0) .
$$




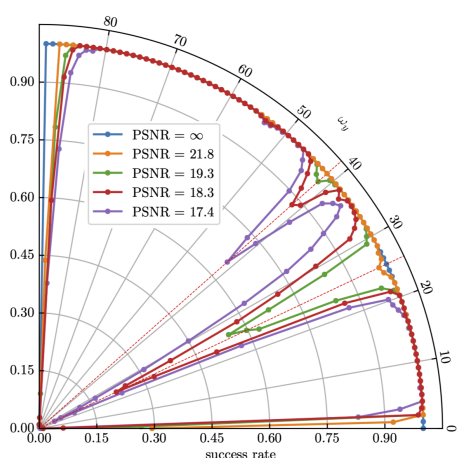

(a)
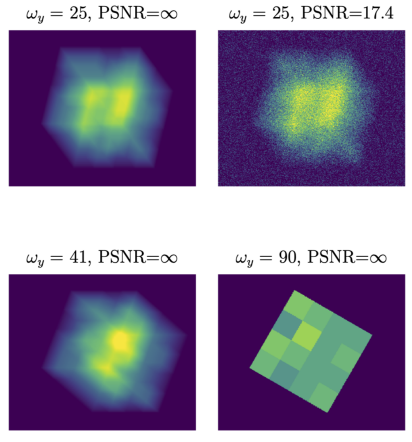

(b)
Fig. 14. Orthogonal Projection: (a) Success Rate in bit reconstruction vs. scan angle $\omega_{y}$. (b) Scans at angles that the success rate is low (shown by dashed lines in (a)). For $\omega_{y}=25$, a noisy scan example at PSNR $=17.4$ is shown.

Then, we define the scan PSNR as

$$
10 \log _{10} \frac{\max _{i, j}\left(Q\left(x_{i}, y_{j} ; T\right)\right)^{2}}{\frac{1}{N_{x} N_{y}} \sum_{i}^{N_{x}} \sum_{j}^{N_{y}}\left(Q\left(x_{i}, y_{j} ; T\right)-\widetilde{Q}\left(x_{i}, y_{j} ; T\right)\right)^{2}},
$$

where $Q\left(x_{i}, y_{j} ; T\right)$ is the sample value of each point of an $N_{x} \times N_{y}$ sampling grid. For each experiment, we try $\sigma_{\nu} \in\{0,0.3,0.4,0.45,0.50\}$ and report the average PSNR value over the performed experiments. For each given barcode orientation and noise level, we do 320 trials and compute the ratio of instances for which we successfully retrieve all the original 48 information bits correctly. We call this ratio the success rate.

1) Orthogonal Projection: Recall that for orthogonal projection, the value of $\omega_{z}$ does not affect the scan values. Thus, we set it to zero in this experiment. We also assume that $\omega_{x}=30^{\circ}$ and change the value of $\omega_{y}$ and noise as explained above. In Figure 14a we show the success rate as a function of $\omega_{y}$, with each color representing a different noise level. We can see that in particular, for four values of $\omega_{y} \in\left\{0^{\circ}, 25^{\circ}, 41^{\circ}, 90^{\circ}\right\}$ the reconstruction becomes difficult in the presence of large noise. Let us take a closer look at some of these angles in Figure 14p. For $\omega_{y}=25^{\circ}$, the barcode orientation is such that the projection of the front and back edges align with each other, causing a mirror symmetry in the scan around the upper-left and lower-right diagonal of the scan. For $\omega_{y}=41^{\circ}$, we see a similar case with a mirror symmetry around the upper-right and lower-left diagonal. At $\omega_{y}=90^{\circ}$, we lose information about the order of different layers of the barcode. Thus, the algorithm fails to reconstruct the bits, even in the absence of noise. Beyond these difficult orientations, we see that the algorithm performs well, even in the presence of large noise, for $\omega_{y} \in\left[4^{\circ}, 16^{\circ}\right] \cup\left[47^{\circ}, 80^{\circ}\right]$. To give a visual indication of the noise level, we also show an example of a noisy scan corresponding to $\omega_{y}=25^{\circ}$ and PSNR $=17.4$ in Figure 14p.

2) Central Projection: In central projection, we have six free parameters to set. Since this results in a very large search grid, we restrict this study to $\boldsymbol{\omega}=\left[30^{\circ}, w_{y}, 40^{\circ}\right]^{\top}$ and $\boldsymbol{t}=[-2,-2,10]^{\top}$. We also assume that $z_{s}=25 \mathrm{~cm}$. The above values are chosen arbitrarily. In Figure 15, we show the success rate for different values of $\omega_{y}$ and noise levels. We see that for three values $\omega_{y} \in\left\{8^{\circ}, 28^{\circ}, 81^{\circ}\right\}$ the success rate

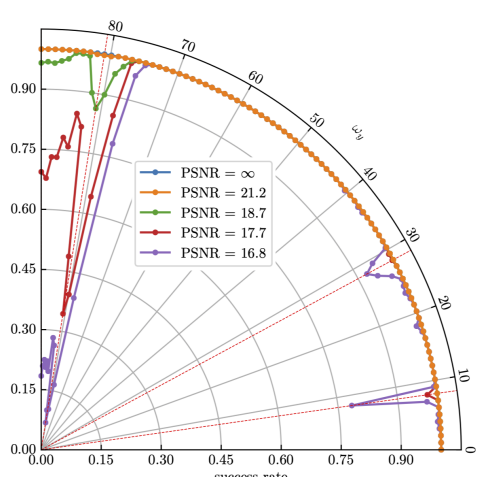

(a)
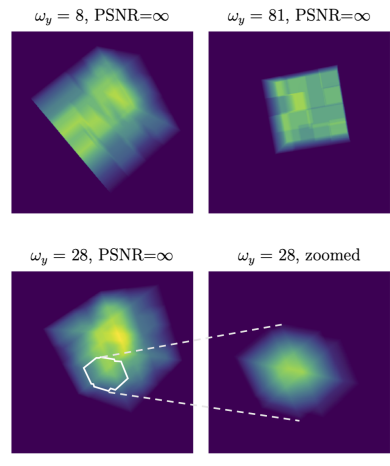

Fig. 15. Central Projection: (a) Success Rate in bit reconstruction vs. scan angle $\omega_{y}$. (b) Scans at angles that the success rate is low (shown by dashed lines in (a)). For $\omega_{y}=28$, we also show on the bottom-right the zoomed scan of three bits that align in such a way that causes a confusion for the reconstruction, and thus a reduction in the success rate.

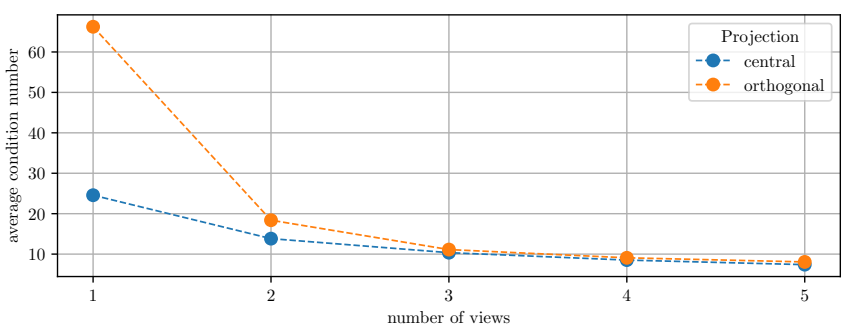

Fig. 16. Average condition number of $\boldsymbol{L}_{\text {stk }}$ vs. the number of views $P$.

deteriorates for large noise perturbations. In Figure 15b, we show the scans for these angles. We can see that at $\omega_{y}=8^{\circ}$ and $\omega_{y}=81^{\circ}$ we fall into a special category of central projection where the barcode shadow has only five or four corners, respectively. As the figures suggest, in these special cases, layers of the barcode bits fall behind each other and create a confusion in the order of the reconstructed layers. For $\omega_{y}=28^{\circ}$, explaining the drop in success rate is slightly more complicated. For this specific orientation and translation, bits $(0,1,2),(1,0,0)$ and $(1,0,1)$ make a special arrangement, which is not only symmetric around the diagonal, but also bit $(1,0,1)$ covers the other two bits almost completely, leaving a very small independent region for the other two bits. Thus, a reduction in the value of one bit is compensated by an increase in the value of the other two. The mistakes in the reconstruction of these three bits cause the lower success rate for this barcode orientation shown in Figure 15a. We show the zoomed scan of these three bits in the bottom-right image in Figure 15p. Again, for the majority of cases, our algorithm performs well, even in the case of large noise.

\section{Effect of Multiple Projections on Stability}

Throughout this paper, we considered the most challenging case where the number of projections is 1 . However, we know intuitively that increasing the number of projections should (at least on average) improve the stability of the reconstruction algorithm. Assume that we have $P$ projections from $P$ random directions. For each projection, we produce the barcode shadow and estimate the orientation of the barcode. 
Next, we form $\boldsymbol{L}_{\text {stk }}$ and $\boldsymbol{y}_{\text {stk }}$, by vertically stacking all $\boldsymbol{L}_{T}^{(p)}$ and $\boldsymbol{y}^{(p)}$ for $p=1, \cdots, P$ :

$$
\boldsymbol{L}_{\mathrm{stk}}=\left[\boldsymbol{L}_{T}^{(1)^{\top}}, \cdots, \boldsymbol{L}_{T}^{(P)^{\top}}\right]^{\top}, \boldsymbol{y}_{\mathrm{stk}}=\left[\boldsymbol{y}^{(1)^{\top}}, \cdots, \boldsymbol{y}^{(P)^{\top}}\right]^{\top}
$$

Then we solve the following equation system: $\boldsymbol{y}_{\text {stk }}=\boldsymbol{L}_{\text {stk }} \boldsymbol{\mu}$. Note that each $\boldsymbol{L}_{T}^{(p)}$ has 24 equivalent matrices, which result in identical barcode shadows. To deal with this, we consider all $24^{P}$ possible combinations among different views. Among all recovered $\boldsymbol{\mu}$ vectors, only the true code will pass the error correction and parity check in the decoder.

In order to evaluate whether cooperating multiple views offers any additional stability in the reconstruction, we compute the condition number of the stacked matrix $\boldsymbol{L}_{\text {stk }}$ in (14), for different number of projections. For each of the orthogonal and central projection cases, we generated 10 random views. Then we calculated the average condition number when selecting only one, or two, ..., up to five out of these 10 views. For example, when we choose 2 views, the average condition number is calculated over all $\left(\begin{array}{c}10 \\ 2\end{array}\right)$ combinations of views. The result of this simulation is shown in Figure 16 for both central and orthogonal projections. We can see that for both projection types, the average condition number decreases as we increase the number of projections. We can interpret this reduction in the condition number as additional stability in reconstruction. Furthermore, the average condition number is better in the case of central projection than orthogonal projection. This is expected, as central projection results in less symmetry in the projection image compared to the orthogonal projection and therefore we achieve more robustness in the reconstruction.

\section{E. Real Experiment, Central Projection Scenario}

For testing the central projection case, we performed a real experiment. We used the same design that we explained in Section VII-A We used 3-D printers to print the barcode bits using two materials with different X-ray attenuation. Since we did not have access to a printer that would print with two materials simultaneously, we decomposed the barcode design into a 3-D puzzle and printed the parts separately with two different printers. We printed the one bits as a whole structure using ABS-M30 Black material with a "Fortus $400 \mathrm{mc}$ " FDM printer. The zero bits were printed in smaller parts using RC70 material with an "Envisiontec Perfactory 4 Mini XL" DLP printer. We attached the barcode with a $30^{\circ}$ tilt (the bottom side of the barcode cube has a $30^{\circ}$ angle with horizon) on an ABS-M30 stand to produce a non-trivial orientation in the barcode for this experiment. The tilt angle is assumed unknown during the reconstruction procedure. Note that the stand is not part of the barcode and is ignored in the reconstruction process. The printed and assembled barcode is shown in Figure 17a In addition, we provide a video of the barcode construction in the supplementary material. We estimated the X-ray attenuation of ABS-M30 Black and RC70 to be 0.39 and 0.61 , respectively (thus the choice of simulated attenuation values in Section VII-C1).

In order to scan the barcode, we used an "RX-SOLUTIONS Ultratom micro CT" cone-beam scanner with a $160 \mathrm{~kW}$ tube, at a $40 \mathrm{kV}$ voltage and a beam angle of $140^{\circ}$. The setup with

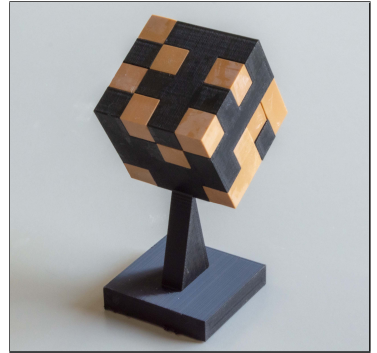

(a)

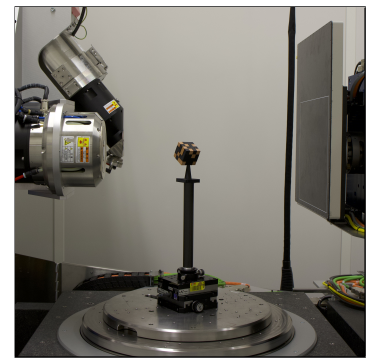

(b)
Fig. 17. Real experiment with central projection scanner. (a) Printed cubic barcode with a $30^{\circ}$ tilt fixed on a stand, (b) cone-beam X-ray scanner with the barcode fixed inside.

the source (on the left), the object and the scan plane (on the right) is shown in Figure 17b For this experiment, we set the source object distance (defined as the distance of the source point to the center of the cylindrical stand where the object is lying) to $20 \mathrm{~cm}$ and the source detector distance to $50 \mathrm{~cm}$. The cylindrical stand in the middle rotates in constant angles (in this experiment $11.25^{\circ}$ ) and at each step an X-ray scan is recorded. This way, we have several scans with different orientations to test our proposed algorithm.

Recall from Section II that the observed values on the scan plane are $I(x, y)$. We need to first transform these values to linear measurements $Q(x, y ; T)$, defined in 13 , as

$Q(x, y ; T)=\ln \left(\frac{I_{0}}{I(x, y)}\right) \approx \ln \left(\max _{x, y} I(x, y)\right)-\ln (I(x, y))$,

where we have used $\max _{x, y} I(x, y)$ as an approximation for $I_{0}$. In the first column of Figure 18, we plot $Q(x, y ; T)$ for four different barcode orientations captured by the X-ray scanner.

In the next step, we need to estimate the values of $\boldsymbol{\omega}$ and $\boldsymbol{t}$. To do so, we follow Steps 1 and 2 from Section III to extract the barcode corners. In this experiment, we manually delete the corners detected for the barcode stand, as in real life this stand will not be present anymore. The second column of Figure 18 shows the extracted corners enclosing the barcode shadow. We then follow Steps 3 to 5 in Section III-B to to estimate the parameters of the barcode pose, $\widehat{\boldsymbol{\omega}}$ and $\boldsymbol{t}$, in the case of central projection. Now that we have the pose parameters, we can also simulate a synthetic scan with those parameters to compare how well its corners match with the extracted corners. As you can see in the third column of Figure 18, the extracted and simulated corners match very well for all the presented orientations. Finally, once the pose parameters are estimated, we can construct matrix $\boldsymbol{L}_{T}$ and vector $\boldsymbol{q}$ in (15) and estimate the attenuation coefficients. We can then use a threshold equal to 0.5 to compute the bits. These attenuation values, together with the ground truth values are shown in the last column of Figure 18. The bits that are estimated wrong are shown in red, amounting to $0,0,1$ and 1 errors from top to bottom. In the next step, we construct all 24 possible arrangements of these bits, according to Section V and pass them through our Reed-Solomon decoder. As the maximum error in all the above cases have been 1 bit, the Reed-Solomon decoder successfully finds and corrects the erroneous bit value, resulting in the 48 original information bits. 

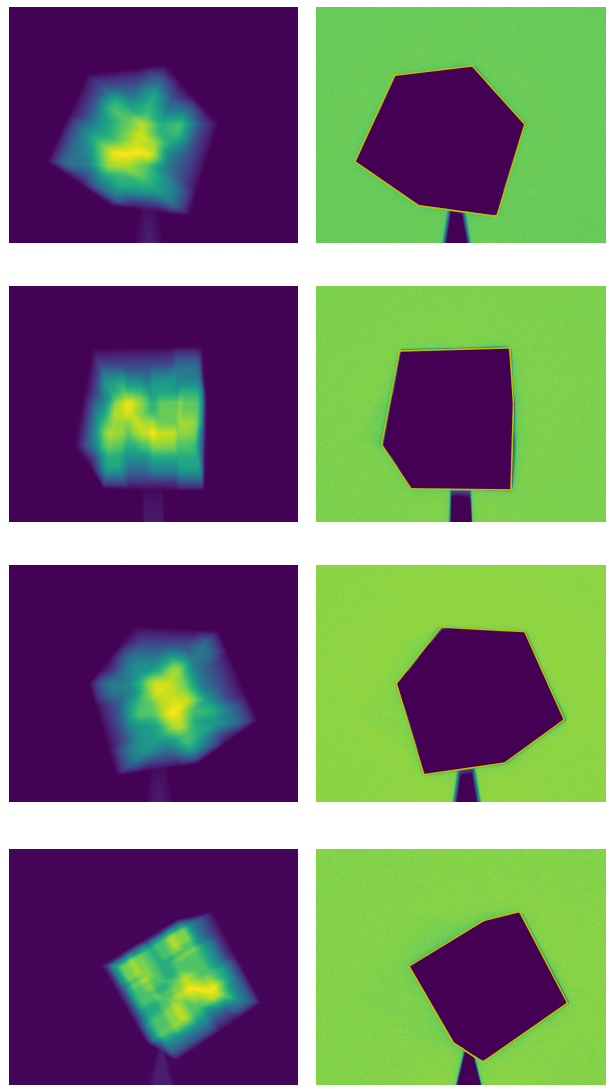
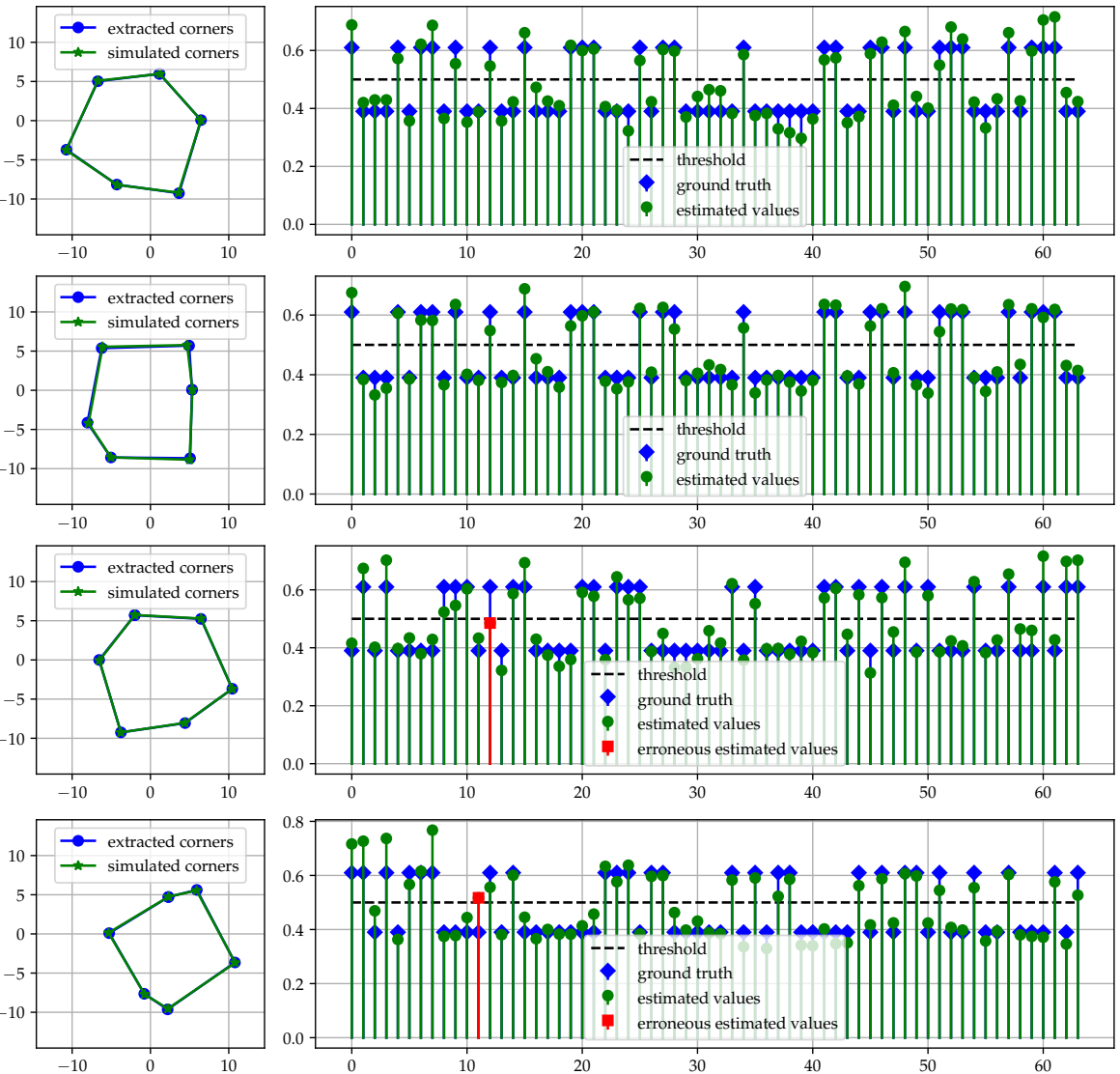

Fig. 18. Results of the real experiment with different barcode orientations in each row. First column: the linear scan measurements $Q(x, y ; T)$, second column: the barcode shadow and the enclosing hexagon constructed by the scan corners, third column: extracted corners, compared to simulated corners computed using estimated orientation parameters $\widehat{\boldsymbol{\omega}}$ and $\widehat{\boldsymbol{t}}$, fourth column: reconstructed attenuation coefficients compared to the ground truth; erroneous reconstructed coefficients are shown in red.

\section{BARcode Materials AND Suitable ENVIRONMENTS}

A great advantage of 3-D barcodes scanned with penetrating waves is that they do not require a direct line of sight with the scanner, in contrast to 2-D barcodes such as QR codes. Thanks to this property, they can be placed or hidden inside other environments and materials. In the above derivations, we ignored the effect of the enclosing environment on the scan of the barcode. In a realistic setup however, we need to also take into account and remove this effect. Thus, the choice of material for building the barcode as well as the environments that the barcode can be utilized become important. In this manuscript, we aimed at providing a proof of concept for showcasing the end-to-end process of coding, implementing, scanning and reconstructing 3-D barcodes. In doing so, the limitations in constructing the barcode with the 3-D printers at hand left us with a limited choice of materials to build the barcode with. In practical implementations, one can choose materials with significantly different attenuation coefficients. For example, with an X-ray with $20 \mathrm{KeV}$ photon energy, polyethylene has a mass attenuation coefficient of 0.43 , while for lead glass, this number is 65.7 [33]. The barcodes need to be built with materials with high mass attenuation and placed in environments with small mass attenuation. The choice of the materials depends on the available technology for constructing and scanning the barcode.

\section{CONClusion}

We introduced 3-D barcodes, which can be read using a single 2-D projection made with penetrating waves such as $\mathrm{X}$-rays, light or sound. We provided a mathematical representation of their attenuation coefficients and showed that if the pose of the barcode in space is known, the reconstruction of the information is as simple as inverting a linear equation. We introduced a unified method for estimating the unknown pose of the barcode using a single 2-D scan, both for parallel-beam and cone-beam scanners. We further proposed coding schemes to correct errors and ambiguities of the reconstruction. Our simulations and real-world implementation of cubic barcodes showed that these objects can be reliably used for carrying information and, by using our algorithms, a single 2-D scan of these barcodes is enough to retrieve their information in the presence of noise.

\section{Acknowledgments}

The authors would like to thank the reviewers of this article for their detailed study of the work, and for providing valuable comments which significantly improved the readability and quality of this paper. In particular, the authors thank one of the reviewers who proposed a geometrical approach for estimating the barcode pose from a single central projection. The reviewer's idea inspired the authors to find a unified 
geometrical approach for solving the pose estimation problem for both orthogonal and central projections.

\section{APPENDIX}

\section{A. Formulation of Line Lengths Matrix}

Recall that for filling the matrix $\boldsymbol{L}_{T}$ (equivalently finding $l_{n}(x, y ; T)$ ), we need to find the intersection of a line passing through point $[x, y, 0]^{\top}$ in direction $\boldsymbol{v}(x, y)$ and the $n$-th cube in the barcode. An intuitive explanation of (12) is that for a given point $[x, y, 0]^{\top}$ on the scan plane, we first transform the coordinate system with the inverse of $T(\cdot), T^{-1}(\cdot)$, and then find the intersection of the lines with the base cube located at the origin. We can find the coordinates of a point on the plane and also the line directions $\boldsymbol{v}^{\prime}$ in the new coordinate system as

$$
\left[\begin{array}{c}
\boldsymbol{x}^{\prime} \\
1
\end{array}\right]=\boldsymbol{T}^{-1}[x, y, 0,1]^{\top}, \quad\left[\begin{array}{c}
\boldsymbol{v}^{\prime} \\
1
\end{array}\right]=\boldsymbol{T}^{-1}\left[\begin{array}{l}
\boldsymbol{v} \\
1
\end{array}\right],
$$

where matrix $\boldsymbol{T}$ is defined in (9). $\boldsymbol{v}$ is defined differently for the orthogonal (OP) and central projections (CP),

$$
\text { OP: } \boldsymbol{v}=[0,0,1]^{\top}, \quad \text { CP: } \boldsymbol{v}=\left[-x,-y, z_{s}\right]^{\top} .
$$

Now we only need to find the intersection of the line $\boldsymbol{x}=$ $\alpha \boldsymbol{v}^{\prime}+\boldsymbol{x}^{\prime}-\boldsymbol{t}_{n}$ with a base cube at the origin with side length equal to $d$, defined by $\psi(\boldsymbol{x})$ according to (5). The line intersects with at most two sides of the base cube. The distance between these two intersection points is $l_{n}(x, y ; T)$ :

$$
l_{n}(x, y ; T)= \begin{cases}\left\|\boldsymbol{x}_{n, i}-\boldsymbol{x}_{n, j}\right\| & \text { if } \exists(i, j) \in \mathcal{J}, \\ 0 & \text { otherwise, }\end{cases}
$$

where, $\boldsymbol{x}_{n, i}$ is the intersection point of the line with the $i$ th side of the $n$-th base code-bit cube. We define the set $\mathcal{J}=\left\{(i, j) \mid i, j \leq 6, i \neq j, 0 \leq \boldsymbol{x}_{n, i} \leq d, 0 \leq \boldsymbol{x}_{n, j} \leq d\right\}$. For example, in Figure 4a, the line intersects $n$-th code-bit cube on the fourth and the fifth sides, therefore $l_{n}(x, y ; T)$ is defined as the distance between $\boldsymbol{x}_{n, 4}$ and $\boldsymbol{x}_{n, 5}$ points. With the same intuition, in Figure $4 \mathrm{~b}, l_{n}(x, y ; T)$ is the distance between points $\boldsymbol{x}_{n, 1}$ and $\boldsymbol{x}_{n, 4}$ illustrated in red. Once we find $l_{n}(x, y ; T)$, we can fill in matrix $\boldsymbol{L}_{T}$ in (14).

\section{B. Proof of Lemma 1}

In order to prove the lemma, we only need to prove that there is a one-to-one relationship between the barcode pose and the barcode shadow (up to the trivial equivalence classes). Once we prove this property, then having a full-rank matrix $\boldsymbol{L}_{T}$ results in a unique reconstruction of the code bits. The ambiguity in the code orientation in the barcode will give us the result up to a permutation out of the given 24 cases shown in Figure 6 ,

a) Orthogonal Projection: Consider the notation in Figure $7 \mathrm{k}$. Take the three shadow corners $p_{1}, p_{2}$ and $p_{3}$, corresponding to barcode vertices $v_{1}, v_{2}$ and $v_{3}$. Each of the vertices $v_{1}, v_{2}$ and $v_{3}$ need to be placed on the line orthogonal to $\mathcal{P}$ at $p_{1}, p_{2}$ and $p_{3}$, respectively. Since the translation of the barcode in the $z$-direction does not change the scan, let us fix $v_{1}$ somewhere on its corresponding line. Given the known length of edges $v_{1}-v_{2}$ and $v_{1}-v_{3}$, which are equal to $N d$, we can have only two possible positions for each of $v_{2}$ and $v_{3}$. This results in four combinations for the locations of these two vertices. But it is easy to see that only two of these combinations result in edges $v_{1}-v_{2}$ and $v_{1}-v_{3}$ be orthogonal to each other: one resulting in the pose shown in Figure 7 and the other one to its mirror against a plane parallel to $\mathcal{P}$. Both of these poses produce the same matrix $\boldsymbol{L}_{T}$ and thus we can reconstruct the barcode bits.

b) Central Projection: Consider the notation in Figure $7 \mathrm{p}$. We saw that the cross product of $\boldsymbol{n}_{1,3}$ and $\boldsymbol{n}_{6,8}$ gives the unique direction of the two barcode edges $v_{1}-v_{3}$ and $v_{6}-v_{8}$. The length of these edges is known (equal to $N d$ ). Moreover, the barcode vertices $v_{1}, v_{3}, v_{6}$, and $v_{8}$ need to placed on lines $s-p_{1}, s-p_{3}, s-p_{6}$ and $s-p_{8}$ respectively. Hence, we have a unique placement of these points in space. With this, we have the two opposite cube edges $v_{1}-v_{3}$ and $v_{6}-v_{8}$ uniquely placed in space. We can find only a single cube that has these edges as its opposite edges. Thus, the given barcode shadow corresponds to a unique barcode pose in space. In the case of the special class of barcode poses shown in Figure 8 , we have seen how to identify the shadow edges that correspond to parallel edges of the barcode. Once this is done, the same arguments as above follow.

\section{Proof of Lemma 2}

Consider a bit region over the scan plane as illustrated in Figure 10 a in blue. For an arbitrary scan direction, the bit region of a cubic voxel is a convex polygon. In order to guarantee at least one sample from inside this bit region, we need to find a maximum distance between samples such that at least one sample falls inside any rotation and translation of each bit region. We define the inscribed circle $\mathcal{C}\left(\omega_{x}, \omega_{y}\right)$ with radius $d r_{c}\left(\omega_{x}, \omega_{y}\right)$ as the the largest circle that can fit inside the bit region. The inscribed square of $\mathcal{C}\left(\omega_{x}, \omega_{y}\right)$ is illustrated as $\mathcal{S}_{\mathcal{C}}\left(\omega_{x}, \omega_{y}\right)$ and it shows the largest square inside the circle. The four corner points of $\mathcal{S}_{\mathcal{C}}\left(\omega_{x}, \omega_{y}\right)$ are the worst case of sampling positions that still lie on the edges of the polygon. The side length of square $\mathcal{S}_{\mathcal{C}}\left(\omega_{x}, \omega_{y}\right)$ is $d \sqrt{2} r_{c}\left(\omega_{x}, \omega_{y}\right)$. With any arbitrary rotation, $\mathcal{S}_{\mathcal{C}}\left(\omega_{x}, \omega_{y}\right)$ will remain inside the polygon. We set the maximum distance between these 4 corner points (diagonal) to be less than minimum distance between parallel sides of bit region. Now, if the samples are taken over the $x$-axis and $y$-axis with spacing $D_{x}, D_{y} \leq d \sqrt{2} r_{c}\left(\omega_{x}, \omega_{y}\right)$, then with any rotation of scan plane or translation, at least one sample lies inside this bit region. The rotated sampling grid is shown in Figure 10a. Note that as expected, through $r_{c}\left(\omega_{x}, \omega_{y}\right)$, the maximum distance between samples depends on the angle from which the scan is taken. The value of $r_{c}\left(\omega_{x}, \omega_{y}\right)$ is depicted in Figure $10 \mathrm{p}$ as a function of $\omega_{x}$ and $\omega_{y}$.

\section{REFERENCES}

[1] A. J. Batista, P. G. Vianna, H. B. Ribeiro, C. J. S. de Matos, and A. S. L. Gomes, "QR code micro-certified gemstones: femtosecond writing and raman characterization in diamond, ruby and sapphire," Scientific Reports, vol. 9, 2019.

[2] A. Kuznetsov, O. Stefanovych, Y. Gorbenko, O. Smirnov, V. Krasnobaev, and K. Kuznetsova, "Information hiding using 3D-printing technology," in IEEE International Conf. on Intelligent Data Acquisition and Advanced Computing Systems, vol. 2, 2019, pp. 701-706. 
[3] B. Silver and N. J. Woodland, "Classifying apparatus and method," U.S. Patent US2612994A, Oct. 1952.

[4] F. H. Stites and A. Raymond, "Mark sensing," U.S. Patent US3225177A, Dec. 1965.

[5] D. Allais, "Multitrack bar code and associated decoding method," U.S. Patent US4794239A, Dec. 1988.

[6] "Automatic identification and data capture techniques - data matrix bar code symbology specification, iso/iec 16022," 2006.

[7] A. Longacre, Jr. and R. Hussey, "Two dimensional data encoding structure and symbology for use with optical readers," U.S. Patent US5591956A, Jan. 1997.

[8] Y. P. Wang, "System for encoding and decoding data in machine readable graphic form," U.S. Patent US5243655A, Sep. 1993.

[9] M. Hara, M. Watabe, T. Nojiri, T. Nagaya, and Y. Uchiyama, "Optically readable two-dimensional code and method and apparatus using the same," U.S. Patent US5726435A, Mar. 1998.

[10] M. R. Miller, G. P. Miller, and R. N. Bernstein, "System and method for providing electronic information upon receipt of a scanned bar code," Patent WO2000065509, Nov. 2000

[11] M. Rohs, "Real-world interaction with camera phones," in Ubiquitous Computing Systems, H. Murakami, H. Nakashima, H. Tokuda, and M. Yasumura, Eds. Springer Berlin Heidelberg, 2005, pp. 74-89.

[12] H. Kato and K. T. Tan, "Pervasive 2D barcodes for camera phone applications," IEEE Pervasive Computing, vol. 6, no. 4, pp. 76-85, 2007.

[13] T.-d. Han, C.-h. Cheong, N.-k. Lee, and E.-d. Shin, "Machine readable code image and method of encoding and decoding the same," U.S. Patent US7020327, Mar. 2006.

[14] "New system to combat online banking fraud," 2013 (Accessed 10 Aug. 2020). [Online]. Available: https://www.cam.ac.uk/research/news/ new-system-to-combat-online-banking-fraud

[15] Microsoft Research, "High capacity color barcodes (HCCB)," 2007 (Accessed 10 Aug. 2020). [Online]. Available: https://www.microsoft. com/en-us/research/project/high-capacity-color-barcodes-hccb

[16] "Jab code (just another bar code)," 2017 (Accessed 10 Aug. 2020). [Online]. Available: https://github.com/jabcode/jabcode

[17] R. F. Eliud, B. A. J. Robert, and P. F. Timothy, "Method and system for generating and printing three dimensional barcodes," U.S. Patent US20160260001, Jan. 2018.

[18] P. Prasad, "Re-writable two-photon three dimensional optical memory," State University of New York at Albany, Tech. Rep., 2001.

[32] G. Tan and T. Herfet, "Application layer hybrid error correction with Reed-Solomon code for DVB services over wireless LANs," in 2007
[19] R. Kikuchi, S. Yoshikawa, P. K. Jayaraman, J. Zheng, and T. Maekawa, "Embedding QR codes onto B-spline surfaces for 3D printing," Computer-Aided Design, vol. 102, pp. 215-223, 2018.

[20] D. F. Swinehart, "The Beer-Lambert law," Journal of Chemical Education, vol. 39, no. 7, p. 333, 1962.

[21] J. Baruchel, J. Y. Buffiere, and E. Maire, X-ray tomography in material science. Hermes science publications, 2000.

[22] E. Maire and P. J. Withers, "Quantitative X-ray tomography," International Materials Reviews, vol. 59, no. 1, pp. 1-43, 2014.

[23] W. A. Kalender, "X-ray computed tomography," Physics in Medicine and Biology, vol. 51, no. 13, pp. R29-R43, jun 2006.

[24] B. D. Smith, "Cone-beam tomography: recent advances and a tutorial review," Optical Engineering, vol. 29, no. 5, pp. 524-534, 1990.

[25] S. G. Chang, Bin Yu, and M. Vetterli, "Adaptive wavelet thresholding for image denoising and compression," IEEE Transactions on Image Processing, vol. 9, no. 9, pp. 1532-1546, 2000.

[26] C. Harris and M. Stephens, "A combined corner and edge detector," in In Proceedings of Fourth Alvey Vision Conference, 1988, pp. 147-151.

[27] I. S. Reed and G. Solomon, "Polynomial codes over certain finite fields," Journal of the Society for Industrial and Applied Mathematics, vol. 8, no. 2, pp. 300-304, 1960.

[28] T. Arai, H. Okamoto, K. Nishimura, M. Kobayashi, and T. Takeuchi, "High capability error correction LSI for CD player and CD Rom," IEEE Trans. on Consumer Electronics, vol. 30, no. 3, pp. 353-359, 1984.

[29] H. C. Chang and C. Shung, "A Reed-Solomon Product-Code (RSPC) decoder for DVD applications," in IEEE International Solid-State Circuits Conference, 1998, pp. 390-391.

[30] P. Kieseberg, M. Leithner, M. Mulazzani, L. Munroe, S. Schrittwieser, M. Sinha, and E. Weippl, "QR code security," in Proceedings of the 8th International Conference on Advances in Mobile Computing and Multimedia, 2010, pp. 430-435.

[31] P. Golden, H. Dedieu, and K. Jacobsen, Fundamentals of DSL Technology. Auerbach Publications, 2006.

International Conference on Wireless Communications, Networking and Mobile Computing, 2007, pp. 2952-2955.

[33] J. H. Hubbell and S. M. Seltzer, "Tables of X-ray mass attenuation coefficients and mass energy-absorption coefficients $1 \mathrm{kev}$ to $20 \mathrm{mev}$ for elements $\mathrm{z}=1$ to 92 and 48 additional substances of dosimetric interest," 5 1995. [Online]. Available: https://www.osti.gov/biblio/76335 